\title{
Monopole operators and symmetry enhancement in ABJM theory revisited
}

\author{
Shan $\mathrm{Hu} \odot^{*}$ \\ Department of Physics, Faculty of Physics and Electronic Sciences, Hubei University, \\ Wuhan 430062, People's Republic of China
}

(Received 15 October 2021; accepted 14 November 2021; published 27 December 2021)

\begin{abstract}
We construct monopole operators for 3D Yang-Mills matter theories and Chern-Simons matter theories in canonical formalism. In this framework, monopole operators, although they are disorder operators, could be written in terms of the fundamental fields of the theory, and thus could be treated in the same way as the ordinary operators. We study the properties of the constructed monopole operators. In Chern-Simons matter theories, monopole operators transform as the local operators with the classical conformal dimension 0 under the action of the dilation and are also covariantly constant. In supersymmetric Chern-Simons matter theories like the ABJM model, monopole operators commute with all of the supercharges, and thus are SUSY invariant. The ABJM model with level $k=1,2$ is expected to have enhanced $S O(8)$ R symmetry due to the existence of the conserved extra R-symmetry currents $j_{\mu}^{A B}$ involving monopoles. With the explicit form of the monopole operators given, we prove the current conservation equation $\partial^{\mu} j_{\mu}^{A B}=0$ using the equations of motion. We also compute the extra $\mathcal{N}=2$ supercharges, derive the extra $\mathcal{N}=2$ SUSY transformation rules, and verify the closure of the $\mathcal{N}=8$ supersymmetry.

DOI: 10.1103/PhysRevD.104.125018
\end{abstract}

\section{INTRODUCTION AND SUMMARY}

In any $3 \mathrm{D}$ gauge theory with the gauge group containing a $U(1)$ factor, there is a current $J^{\mu}=\frac{1}{4 \pi} \epsilon^{\mu \nu \lambda} \operatorname{tr} F_{\nu \lambda}$ whose conservation is equivalent to the Bianchi identity. The conserved topological charge

$$
Q=\frac{1}{4 \pi} \int d^{2} x \epsilon^{i j} t r F_{i j}
$$

is called the vortex charge, and the related global symmetry is referred to as the $U(1)_{J}$ symmetry. Monopole operators are local operators creating (annihilating) the charge $Q$ $[1,2]$. As disorder operators, monopole operators cannot be written as the polynomials of the elementary fields at the insertion point, but dualities can map them onto the operators of that kind [3-9]. It is expected that a better understanding of monopole operators can make the 3D dualities more transparent. One such example is the $2 \mathrm{D}$ duality relating the massive Thirring model and the sineGordon model $[10,11]$. In the sine-Gordon model, local disorder operators for the creation and annihilation of

\footnotetext{
*hushan@hubu.edu.cn
}

Published by the American Physical Society under the terms of the Creative Commons Attribution 4.0 International license. Further distribution of this work must maintain attribution to the author(s) and the published article's title, journal citation, and DOI. Funded by SCOAP. topological solitons (kinks) are constructed in canonical formalism, while by rewriting the sine-Gordon model in terms of these dual variables, the massive Thirring model is obtained [11]. In this paper, we will give a similar construction for monopole operators in 3D, although the main concern is not duality.

Monopole operators also play an important role in membrane models, where the topological charge corresponds to the Kaluza-Klein (KK) momentum along the M-theory circle [12-21]. In Ref. [12], Aharony, Bergman, Jaeris, and Maldacena $(\mathrm{ABJM})$ proposed an $\mathcal{N}=6 U(N) \times$ $U(N)$ level $(k,-k)$ Chern-Simons matter theory to describe the low energy dynamics of $N$ coincident $M 2$-branes probing a $\mathbb{C}^{4} / \mathbb{Z}_{k}$ singularity. The theory has the manifest $S U(4) \times U(1)_{J}$ global symmetry, the isometry of $\mathbb{C}^{4} / \mathbb{Z}_{k}$, which is expected to be enhanced to $S O(8)$ when $k=1,2$ [12]. In AdS/CFT correspondence, the ABJM model is dual to $\mathrm{M}$ theory on an $\mathrm{AdS}_{4} \times S^{7} / \mathbb{Z}_{k}$ background. With $S^{7} / \mathbb{Z}_{k}$ viewed as a Hopf fibration over $\mathbb{C P}^{3}$, supergravity modes carrying KK momenta along the fiber circle should be mapped onto the BPS operators involving monopoles $[12,22]$. In particular, when $k=1,2$, twelve dimension-2 currents can be constructed from monopole operators, which are conserved by virtue of the dimensions and would enhance the global symmetry to $S O(8)$ [12]. Such expectation is verified in Ref. [23]. The enhanced $\mathcal{N}=8$ supersymmetry is also studied in Refs. [24-26].

Monopole operators are usually defined by specifying the singularities of the gauge fields at the insertion point and expanding the quantum fields around this singular 
background [2]. For example, in a $U(N)$ gauge theory, the gauge field singularity is supposed to have the form

$$
A_{N / S}=\frac{H}{r}( \pm 1-\cos \theta) d \varphi
$$

for the north and south charts so that there is some magnetic flux on $S^{2}$ surrounding it. $H$ must satisfy the quantization condition $e^{4 \pi i H}=I$ and is an element of the Cartan subalgebra of the form $H=\operatorname{diag}\left(\frac{m_{1}}{2}, \ldots, \frac{m_{N}}{2}\right), m_{i} \in \mathbb{Z}$ [27]. For a CFT on $S^{2} \times \mathbb{R}$, with the specified magnetic flux on $S^{2}$ given, one can compute quantum numbers like the $\mathrm{R}$ charges and energies of the states, which, by stateoperator correspondence, are mapped onto the vortexcharged operators $[2,4,5]$. In this way, $R$ charges and conformal dimensions of the vortex-charged operators can be obtained with the quantum corrections taken into account. Since the method only applies for the weakly coupled theories, some strategies must be used to get the strong-coupling result in ABJM theory [22,23,28].

In this paper, we will return to 't Hooft's original definition of the monopole operator as a singular gauge transformation acting on states [1]. In canonical formalism, such operators can be written in terms of the fundamental variables of the theory, and thus could be treated in the same way as the ordinary operators. This is similar to Ref. [11], where the kink operator is constructed in a 2D sine-Gordon model. In Yang-Mills matter theories and Chern-Simons matter theories, we will give the explicit canonical expressions for the monopole operator $M_{R}(x)$, which is labeled by the representation $R$ of the gauge group and is realized as a gauge transformation singular at $x$. The singular gauge transformation $M_{R}(x)$, although it is labeled by $R$, does not transform in the representation $R$. In ChernSimons matter theories, we will define a dressed monopole operator $\mathcal{M}_{R}(x)$ that would behave as a local operator in the representation $R$ under the local gauge transformation. In non-Abelian gauge theories, $\mathcal{M}_{R}(x)$ is the monopole operator that is used to build the vortex-charged gauge invariants.

We also study the properties of the constructed monopole operators. We prove the contraction relation given in Eq. (5.27), which is required in ABJM theory for R charges to form the so(8) algebra. We show that in Chern-Simons matter theories, under the action of the dilation, monopole operators transform as the local operators with the conformal dimension 0 - which, of course, may get quantum corrections from the interactions. We compute the supersymmetry transformation of the monopole operators in ABJM theory. It turns out that monopole operators are invariant under the $\mathcal{N}=6$ supersymmetry, as well as the $\mathcal{N}=8$ supersymmetry, when $k=1,2$. It seems that in supersymmetric Chern-Simons matter theories, monopole operators always commute with the supercharges, which is not a surprise, since monopole operators are just singular gauge transformations, while the ordinary gauge transformations commute with all gauge-invariant operators. On the other hand, in supersymmetric Yang-Mills matter theories, monopole operators are not SUSY invariant. With the suitable scalar fields added, one may construct BPS scalar-dressed monopole operators preserving part of the supersymmetry.

In ABJM theory, the gauge-invariant combination of the monopole operators and the matter fields gives a new set of local operators carrying the vortex charge. In AdS/CFT, part of the KK modes of supergravity on $\mathrm{AdS}_{4} \times S^{7} / \mathbb{Z}_{k}$ are mapped onto the vortex-charged operators [12]. Since the monopole operators are SUSY invariant and have the classical conformal dimension 0 , these operators could be $1 / 2$ BPS and thus have the protected conformal dimension matching the spectrum on the gravity side.

In the pure Abelian Chern-Simons theory and the $U(1)_{k} \times U(1)_{-k}$ ABJM theory, monopole operators can be written as the Wilson lines ending at the inserting point and are covariantly constant [12,24,29-31]. However, when the charged matter is included or the gauge groups are non-Abelian, monopole operators will not be Wilson lines anymore $[12,26]$. There is also a controversy on their covariant constancy. In Refs. [24,25], the covariant constancy condition for monopole operators was assumed when deriving the $\mathcal{N}=8$ supersymmetry transformation rules in ABJM theory. Meanwhile, in Ref. [26], it was shown that such a condition would lead to Eq. (6.11), a severe constraint on monopole operators and the scalar fields. In fact, even in Ref. [24], for the $S O(8)$ invariant trial Bagger-Lambert-Gustavsson (BLG) Lagrangian to be identical to the ABJM Lagrangian, the algebraic identities given by Eq. (2.7) should also hold, while the closure of the $\mathcal{N}=$ 8 supersymmetry requires a few more similar identities. These identities are all constraint equations relating monopole operators and the matter fields.

In ABJM theory, monopole operators commute with the supercharges, while the anti-commutator of supercharges gives either a covariant derivative or a fielddependent gauge variation, so from Eq. (9.27), one may conclude that monopole operators are both covariantly constant and invariant under that gauge variation. The latter property could be stated as the algebraic identities for monopole operators and the matter fields. This argument is based on super-symmetry. In Sec. VIII, we will give a proof for the covariant constancy of monopole operators in generic Chern-Simons matter theories without relying on supersymmetry. We will also show that in Yang-Mills matter theories, monopole operators are not covariantly constant.

With the explicit form of the monopole operators given, the global symmetry enhancement is revisited. In 3D gauge theories, if the original global symmetry algebra is $\mathcal{R}$, with the $U(1)_{J}$ charge $Q$ added, one may consider the possibility for the enlarged symmetry $\hat{\mathcal{R}}=\mathcal{R} \oplus Q \oplus \mathcal{R}_{\text {off-diag, where }}$ 
$\mathcal{R}_{\text {off-diag }}$ denotes off-diagonal elements charged under $Q$. The form of the global symmetry currents is entirely determined by the field content. The current for $\mathcal{R}_{\text {off-diag }}$ must involve monopole operators and does not always exist. In the $k=1,2 \mathrm{ABJM}$ theory, $\mathcal{R}$ and $\hat{\mathcal{R}}$ are $s u(4)$ and so(8) algebras with the currents for $\mathcal{R}$ and $\mathcal{R}_{\text {off-diag }}$ given by Eqs. (9.1) and (9.3). We compute the current conservation equation $\partial^{\mu} j_{\mu}^{A B}=0$ with the equations of motion plugged in. It turns out that, for $\partial^{\mu} j_{\mu}^{A B}=0$, monopole operators should satisfy the constraints in Eq. (9.10). The first of these is the covariant constancy condition. The rest are the invariance condition under some particular fielddependent gauge variations [stronger than that in Eq. (9.27)], which is also the origin of the algebraic identities in Refs. [24,26]. We show that the constructed monopole operators satisfy Eq. (9.10), and so the global symmetry is enhanced to $S O(8)$.

In fact, in a 3D unitary CFT, the dimension-2 currents are always conserved, so to prove the R-symmetry enhancement, it is enough to show that $j_{\mu}^{A B}$ has the conformal dimension 2, which is the approach in Ref. [23]. In our proof of $\partial^{\mu} j_{\mu}^{A B}=0$, conformal invariance or supersymmetry is not the necessary condition. For example, consider a truncated ABJM model with no fermionic fields, where the monopole operators satisfy the first two equations in Eq. (9.10). Among all $S U(4)$ invariant scalar potentials, only the quadratic mass term and the sextic potential in the ABJM model could make $\partial^{\mu} j_{\mu}^{A B}=0$. In this perspective, the symmetry enhancement comes from the classical symmetry of the Lagrangian together with the properties of the monopole operators.

With the extra $\mathrm{R}$ charges added into the $\mathcal{N}=6$ superconformal algebra of the ABJM theory, two additional supercharges are generated. We get the extra $\mathcal{N}=2$ supercharges in Eq. (10.17). For the commutation relation in Eq. (10.16) to take the presented form, the properties of Eq. (9.10) are used again. We write down the extra $\mathcal{N}=2$ SUSY transformation rules, which are similar with those obtained in Ref. [24]. We verify the closure of the $\mathcal{N}=8$ supersymmetry and also discuss the BPS multiplet structure of the theory following the line of Ref. [32].

The rest of the paper is organized as follows. In Sec. II, we define the monopole operator $M_{R}(x)$ as a singular gauge transformation. In Sec. III, we write down the explicit form of $M_{R}(x)$ in Yang-Mills matter theories and Chern-Simons matter theories. In Sec. IV, we construct the dressed monopole operator $\mathcal{M}_{R}(x)$ that could transform in representation $R$. In Sec. V, we derive some contraction relations for $\mathcal{M}_{R}(x)$. In Sec. VI, we compute the classical conformal dimension of $M_{R}(x)$ and $\mathcal{M}_{R}(x)$. In Sec. VII, we calculate the supersymmetry transformation of $M_{R}(x)$ and $\mathcal{M}_{R}(x)$. In Sec. VIII, we compute the derivative and covariant derivative of $M_{R}(x)$ and $\mathcal{M}_{R}(x)$. In Sec. IX, we prove the current conservation equation $\partial^{\mu} j_{\mu}^{A B}=0$ in ABJM theory.
In Sec. X, we study the enhanced $\mathcal{N}=8$ supersymmetry. Section XI gives the conclusion and discussion.

\section{MONOPOLE OPERATOR AS A SINGULAR GAUGE TRANSFORMATION}

Monopole operators were first introduced by 't Hooft to define an alternative criterion for confinement [1]. The basic idea was to define an operator that creates or annihilates topological charges. It is known that solitons are pure gauge configurations singular at their locations, so the soliton operators can be constructed as the gauge transformations singular at the insertion points. The generic relation between solitons and soliton operators is given in Appendix A. Monopole operators in Ref. [1] can also have the nontrivial winding $n$, which excludes the existence of quarks. We will only consider the situation with $n=0$, so that the action of the monopole operators on fields in the (anti)fundamental representation is also well defined.

Let us start with a brief introduction on group theory. For a group $G$ with the $\operatorname{rank} r,\left\{t_{M} \mid M=1,2, \ldots, \operatorname{dim} G\right\}$ are generators for the Lie algebra of $G$ in the fundamental representation, among which $\left\{H_{A} \mid A=1,2, \ldots, r\right\}$ are generators of the Cartan subalgebra, $\operatorname{tr}\left(t_{M} t_{N}\right)=\frac{1}{2} \delta_{M N}$. Simple coroots are $r$-dimensional vectors $\left\{\vec{\alpha}_{A}^{*} \mid A=\right.$ $1,2, \ldots, r\}$. The irreducible representation $R$ of the group $G$ is labeled by $\vec{m}=\left(m_{1}, \ldots, m_{r}\right)$, with $m_{A} \in \mathbb{Z}, m_{A} \geq 0$, $A=1,2, \ldots, r, m_{1} \geq m_{2} \geq \ldots \geq m_{r}$. When $G$ and the dual group $G^{*}$ are identical such as $G=U(N), R$ is also in oneto-one correspondence with

$$
H_{\vec{m}}=\sum_{A=1}^{r} m_{A} \vec{\alpha}_{A}^{*} \cdot \vec{H}
$$

in Cartan subalgebra, where $\vec{H}=\left(H_{1}, H_{2}, \ldots, H_{r}\right)$, and $\exp \left\{4 \pi i H_{\vec{m}}\right\}=I$. When $G=U(N)$, for an irreducible representation $R$ labeled by $\vec{m}=\left(m_{1}, \ldots, m_{N}\right)$, the corresponding $H_{\vec{m}}$ is

$$
H_{\vec{m}}=\operatorname{diag}\left(\frac{m_{1}}{2}, \ldots, \frac{m_{N}}{2}\right) .
$$

Now, we consider a 3D gauge theory with the canonical coordinate $\left(A_{i}, \Phi\right)$ and the conjugate momentum $\left(\Pi_{i}, \Pi\right)$, where $i=1,2, A_{i}=A_{i}^{M} t^{M}, \Pi_{i}=\Pi_{i}^{M} t^{M}$, and $M=1,2, \ldots$, $\operatorname{dim} G . A_{i}$ is the gauge field in the adjoint representation. $\Phi$ is the matter field in the adjoint or (anti)fundamental representation. The complete orthogonal basis of the Hilbert space can be selected as the eigenstates $\left\{\left|A_{i}, \Phi\right\rangle \mid \forall A, \Phi\right\}$.

In canonical formalism, it is more appropriate to call the monopole operator the vortex operator, since it creates the vortex in 2D space. A vortex at the position $x$ carrying one unit of the vortex charge $Q$ can be descried by the gauge configuration $\frac{1}{2} a_{i}(x, y)$ with 


$$
b(x, y)=\epsilon^{i j} \partial_{i}^{y} a_{j}(x, y)=4 \pi \delta^{2}(x-y),
$$

where by $\partial_{i}^{y}$ we mean the derivative with respect to $y$. The value $a_{i}$ can be solved as

$$
a_{i}(x, y)=2 \epsilon_{j i} \frac{x^{j}-y^{j}}{|x-y|^{2}}+\partial_{i} \sigma(y)
$$

for the arbitrary scalar $\sigma(y)$. Here $a_{i}$ is not a pure gauge, even though one can still construct some $e^{i \omega}$ satisfying

$$
a_{i}(x, y)=-i e^{-i \omega(x, y)} \partial_{i}^{y} e^{i \omega(x, y)}
$$

everywhere except for a singularity at $x$.

The monopole operator $M_{R}(x)$ labeled by $R$ is defined via its action on $\left|A_{i}, \Phi\right\rangle$. When $\Phi$ is in the adjoint representation,

$$
\begin{aligned}
M_{R}(x)\left|A_{i}(y), \Phi(y)\right\rangle= & \mid \Omega_{\vec{m}}(x, y) A_{i}(y) \Omega_{\vec{m}}^{-1}(x, y) \\
& \left.-H_{\vec{m}} a_{i}(x, y), \Omega_{\vec{m}}(x, y) \Phi(y) \Omega_{\vec{m}}^{-1}(x, y)\right\rangle .
\end{aligned}
$$

When $\Phi$ is in the fundamental representation,

$$
\begin{aligned}
M_{R}(x)\left|A_{i}(y), \Phi(y)\right\rangle= & \mid \Omega_{\vec{m}}(x, y) A_{i}(y) \Omega_{\vec{m}}^{-1}(x, y) \\
& \left.-H_{\vec{m}} a_{i}(x, y), \Omega_{\vec{m}}(x, y) \Phi(y)\right\rangle .
\end{aligned}
$$

$\Omega_{\vec{m}}=e^{-i H_{\vec{m}} \omega}$. Moving along a closed curve surrounding $x$, $\omega \rightarrow \omega+4 \pi, \Omega_{\vec{m}} \rightarrow e^{-4 \pi i H_{\vec{m}}} \Omega_{\vec{m}}=\Omega_{\vec{m}} . \Omega_{\vec{m}}$ is single valued, and $e^{-4 \pi i H_{\vec{m}}}=I$ amounts to selecting the winding number $n=0$ in Ref. [1]. Away from $x$,

$$
i \Omega_{\vec{m}}^{-1}(x, y) \partial_{i}^{y} \Omega_{\vec{m}}(x, y)=H_{\vec{m}} a_{i}(x, y)
$$

is satisfied, so $M_{R}(x)$ is a local gauge transformation everywhere except for a singularity at $x$.

\section{MONOPOLE OPERATORS IN CANONICAL FORMALISM}

It is straightforward to write down the operator expression of $M_{R}$ in canonical formalism. We will consider two typical situations: $M_{R}$ in 3D Yang-Mills theory coupling with the matter, and $M_{R}$ in 3D Chern-Simons theory coupling with the matter. Although in these cases, the actions of $M_{R}$ on canonical variables are identical, the explicit forms of $M_{R}$ are different due to the distinct kinetic terms for gauge fields.

In 3D Yang-Mills matter theory, the Gauss constraint is

$$
\Lambda=D_{i} \Pi^{i}-\rho=\partial_{i} \Pi^{i}-i\left[A_{i}, \Pi^{i}\right]-\rho=0,
$$

where $\rho$ is the charge density of the matter fields. Local gauge transformation operator $U(\alpha)$ with the transformation parameter $\alpha$ is given by

$$
U(\alpha)=\exp \left\{-i \int d^{2} y \operatorname{tr}[\alpha(y) \Lambda(y)]\right\}
$$

where $\alpha(y)$ is a Lie-algebra-valued function well defined everywhere. As a singular gauge transformation with the parameter $H_{\vec{m}} \omega, M_{R}(x)$ could be written as

$$
\begin{aligned}
M_{R}(x)= & \exp \left\{-i \int d^{2} y \operatorname{tr}\left[H_{\vec{m}} \omega(x, y) \Lambda(y)\right]\right\} \\
= & \exp \left\{i \int d ^ { 2 } y \operatorname { t r } \left(H _ { \vec { m } } \left[\Pi^{i}(y) a_{i}(x, y)\right.\right.\right. \\
& \left.\left.\left.+i\left[A_{i}(y), \Pi^{i}(y)\right] \omega(x, y)+\rho(y) \omega(x, y)\right]\right)\right\} .
\end{aligned}
$$

In a 3D Chern-Simons matter theory with the level $k$, the canonical commutation relation for the gauge field is [33]

$$
\left[A_{i}^{M}(x), A_{j}^{N}(y)\right]=\frac{2 \pi i}{k} \delta^{M N} \epsilon_{i j} \delta^{2}(x-y),
$$

and the Gauss constraint is

$$
\Lambda=\frac{k}{4 \pi} \epsilon^{i j} F_{i j}-\rho=\frac{k}{2 \pi} \epsilon^{i j}\left(\partial_{i} A_{j}-\frac{i}{2}\left[A_{i}, A_{j}\right]\right)-\rho=0 .
$$

The monopole operator $M_{R}(x)$ is given by

$$
\begin{aligned}
M_{R}(x)= & \exp \left\{-i \int d^{2} y \operatorname{tr}\left[H_{\vec{m}} \omega(x, y) \Lambda(y)\right]\right\} \\
= & \exp \left\{\frac { i k } { 2 \pi } \int d ^ { 2 } y \operatorname { t r } \left(H _ { \vec { m } } \left[\epsilon^{i j} A_{j}(y) a_{i}(x, y)\right.\right.\right. \\
& \left.\left.\left.+\frac{i}{2} \epsilon^{i j}\left[A_{i}(y), A_{j}(y)\right] \omega(x, y)+\frac{2 \pi}{k} \rho(y) \omega(x, y)\right]\right)\right\} .
\end{aligned}
$$

In Eq. (2.4), $a_{i}$ is determined up to a local gauge transformation, $a_{i} \sim a_{i}+\partial_{i} \sigma$. For $M_{R}(x)$ in Eqs. (3.3) and (3.6), with $\omega$ replaced by $\omega+\sigma$, we will get $M_{R}^{\prime}(x) \sim U\left(H_{\vec{m}} \sigma\right) M_{R}(x)$, with $U\left(H_{\vec{m}} \sigma\right)$ being an ordinary local gauge transformation. In particular, in $U(1)$ pure Chern-Simons theory,

$$
M(x)=\exp \left\{\frac{i k}{4 \pi} \int d^{2} y\left[\epsilon^{i j} A_{j}(y) a_{i}(x, y)\right]\right\} .
$$

One can always select the suitable $\sigma$ so that $a_{i}$ is nonvanishing only at a Dirac string $y(s)$ with $0 \leq s<\infty$, $y(0)=x, y(\infty)=\infty$. In this case,

$$
M(x)=\exp \left\{i k \int_{0}^{\infty} d s A_{i}[y(s)] \dot{y}^{i}(s)\right\}
$$


is a Wilson line starting from $x$ and extending to infinity, as expected [29-31].

Similarly to the local gauge transformation, the action of $M_{R}$ on canonical fields is

$$
\begin{aligned}
M_{R}(x) A_{i}(y) M_{R}^{-1}(x)= & e^{i H_{\vec{m}} \omega(x, y)} A_{i}(y) e^{-i H_{\vec{m}} \omega(x, y)} \\
& +H_{\vec{m}} a_{i}(x, y), \\
M_{R}(x) \Pi_{i}(y) M_{R}^{-1}(x)= & e^{i H_{\vec{m}} \omega(x, y)} \Pi_{i}(y) e^{-i H_{\vec{m}} \omega(x, y)},
\end{aligned}
$$

and

$$
M_{R}(x) \Phi(y) M_{R}^{-1}(x)=e^{i H_{\vec{m}} \omega(x, y)} \Phi(y)
$$

or

$M_{R}(x) \Phi(y) M_{R}^{-1}(x)=e^{i H_{\vec{m}} \omega(x, y)} \Phi(y) e^{-i H_{\vec{m}} \omega(x, y)}$

for $\Phi$ in fundamental representation or adjoint representation. However, for the field strength

$$
F=\epsilon^{i j} F_{i j}=\epsilon^{i j}\left(\partial_{i} A_{j}-\partial_{j} A_{i}-i\left[A_{i}, A_{j}\right]\right),
$$

we have

$$
\begin{aligned}
M_{R}(x) F(y) M_{R}^{-1}(x)= & e^{i H_{\vec{m}} \omega(x, y)} F(y) e^{-i H_{\vec{m}} \omega(x, y)} \\
& +8 \pi H_{\vec{m}} \delta^{2}(x-y) .
\end{aligned}
$$

The extra $\delta$ term indicates that $M_{R}(x)$ is a gauge transformation singular at $x$. When $G=U(N)$, for $Q$ given by Eq. (1.1), from Eq. (3.14),

$$
\left[Q, M_{R}(x)\right]=-2 \operatorname{tr} H_{\vec{m}} M_{R}(x),
$$

so $M_{R}(x)$ carries the vortex charge $-2 t r H_{\vec{m}}$.

Finally, for a 3D Chern-Simons matter theory with the gauge group $U(N)_{k} \times U(N)_{-k}$ like the ABJM, supposing that $\hat{A}_{i}$ and $\hat{\rho}$ are the gauge field and charge density for the group $U(N)_{-k}$, the monopole operator $M_{\left[R_{1}, \bar{R}_{2}\right]}(x)$ can be written as

$$
\begin{aligned}
M_{\left[R_{1}, \bar{R}_{2}\right]}(x)= & \exp \left\{\frac{i k}{2 \pi} \int d^{2} y \operatorname{tr}\left(H_{\vec{m}_{1}}\left[\epsilon^{i j} A_{j}(y) a_{i}(x, y)+\frac{i}{2} \epsilon^{i j}\left[A_{i}(y), A_{j}(y)\right] \omega(x, y)+\frac{2 \pi}{k} \rho(y) \omega(x, y)\right]\right)\right\} \\
& \times \exp \left\{-\frac{i k}{2 \pi} \int d^{2} y \operatorname{tr}\left(H_{\vec{m}_{2}}\left[\epsilon^{i j} \hat{A}_{j}(y) a_{i}(x, y)+\frac{i}{2} \epsilon^{i j}\left[\hat{A}_{i}(y), \hat{A}_{j}(y)\right] \omega(x, y)+\frac{2 \pi}{k} \hat{\rho}(y) \omega(x, y)\right]\right)\right\},
\end{aligned}
$$

where $R_{1}$ and $R_{2}$ are two irreducible representations labeled by $H_{\vec{m}_{1}}$ and $H_{\vec{m}_{2}}$, respectively. The Gauss constraint in ABJM implies $\operatorname{tr} F_{i j}=\operatorname{tr} \hat{F}_{i j}$ [12]. For the topological charge

$$
\begin{aligned}
Q^{+} & =\frac{1}{4 \pi} \int d^{2} x \epsilon^{i j} \operatorname{tr}\left(F_{i j}+\hat{F}_{i j}\right) \\
& =\frac{1}{2 \pi} \int d^{2} x \epsilon^{i j} \operatorname{tr} F_{i j}=\frac{1}{2 \pi} \int d^{2} x \epsilon^{i j} \operatorname{tr} \hat{F}_{i j},
\end{aligned}
$$

we have

$$
\begin{aligned}
{\left[Q^{+}, M_{\left[R_{1}, \bar{R}_{2}\right]}(x)\right] } & =-2 \operatorname{tr}\left(H_{\vec{m}_{1}}+H_{\vec{m}_{2}}\right) M_{\left[R_{1}, \bar{R}_{2}\right]}(x) \\
& =-4 \operatorname{tr} H_{\vec{m}_{1}} M_{\left[R_{1}, \bar{R}_{2}\right]}(x) \\
& =-4 \operatorname{tr} H_{\vec{m}_{2}} M_{\left[R_{1}, \bar{R}_{2}\right]}(x),
\end{aligned}
$$

with $\operatorname{tr} H_{\vec{m}_{1}}=\operatorname{tr} H_{\vec{m}_{2}}$ assumed.

\section{MONOPOLE OPERATORS IN REPRESENTATION $\boldsymbol{R}$}

The monopole operator $M_{R}(x)$ discussed in Secs. II and III, although it is labeled by $R$, does not transform in representation $R$ under the gauge transformation. In the following, we will compute the gauge transformation of $M_{R}(x)$ explicitly.

First, in 3D electromagnetic theory coupling with the matter,

$M(x)=\exp \left\{\frac{i}{2} \int d^{2} y\left[\Pi^{i}(y) a_{i}(x, y)+\rho(y) \omega(x, y)\right]\right\}$,

which is invariant under the $U(1)$ gauge transformation.

In $3 \mathrm{D} U(1)$ Chern-Simons theory coupling with the matter,

$$
M(x)=\exp \left\{\frac{i k}{4 \pi} \int d^{2} y\left[\epsilon^{i j} A_{j}(y) a_{i}(x, y)+\frac{2 \pi}{k} \rho(y) \omega(x, y)\right]\right\} .
$$

Under the $U(1)$ local gauge transformation, $A_{i} \rightarrow A_{i}+$ $\partial_{i} \alpha, \rho \rightarrow \rho$,

$$
M(x)=e^{i k \alpha(x)} M(x) .
$$

$M(x)$ transforms as an operator at $x$ carrying the $U(1)$ charge $k$.

In 3D non-Abelian gauge theory with the group $G$, suppose that $\mathcal{G}$ is the group composed by the local gauge transformation operator $U, \forall U \in \mathcal{G}$ : 


$$
\begin{aligned}
& U A_{i} U^{-1}=u^{+} A_{i} u+i u^{+} \partial_{i} u, \\
& U \Pi_{i} U^{-1}=u^{+} \Pi_{i} u, \quad U \rho U^{-1}=u^{+} \rho u,
\end{aligned}
$$

where $u \in G$ is the transformation matrix for $U$. Concretely, for $U(\alpha)$ given by Eq. (3.2), the related $u(y)=e^{-i \alpha(y)}$. Actions of $U$ on $M_{R}(x)$ in Eqs. (3.3) and (3.6) are given by

$$
U M_{R}(x) U^{-1}=\exp \left\{i \int d^{2} y \operatorname{tr} H_{\vec{m}}\left[u^{+}\left(\Pi^{i} a_{i}+i\left[A_{i}, \Pi^{i}\right] \omega+\rho \omega\right) u-u^{+}\left[\partial_{i} u u^{+}, \Pi^{i}\right] u \omega\right]\right\}
$$

and

$$
\begin{aligned}
U M_{R}(x) U^{-1}= & \exp \left\{\frac { i k } { 2 \pi } \int d ^ { 2 } y \operatorname { t r } ( u H _ { \vec { m } } u ^ { + } ) \left[\left(\epsilon^{i j} A_{j} a_{i}+\frac{i}{2} \epsilon^{i j}\left[A_{i}, A_{j}\right] \omega+\frac{2 \pi}{k} \rho \omega\right)\right.\right. \\
& \left.\left.+i \epsilon^{i j}\left[A_{i}, i \partial_{j} u u^{+}\right] \omega+\frac{i}{2} \epsilon^{i j}\left[i \partial_{i} u u^{+}, i \partial_{j} u u^{+}\right] \omega+i \epsilon^{i j} a_{i} \partial_{j} u u^{+}\right]\right\},
\end{aligned}
$$

respectively. Obviously, $M_{R}(x)$ does not transform in the representation $R$.

It is desirable to construct the monopole operator that would transform as a local operator in the representation $R$ under the action of $U$. Suppose that $\{|\alpha\rangle \mid \alpha=$ $1,2, \ldots, \operatorname{dim} R\}$ are bases for the representation $R$, among which $|1\rangle$ is the highest weight state. The group element $u$ in the representation $R$ is given by $D_{\beta R}^{\alpha}(u)=\langle\alpha|u| \beta\rangle$, and $D_{\gamma R}^{\alpha}(u v)=D_{\beta R}^{\alpha}(u) D_{\gamma R}^{\beta}(v)$. Consider

$$
\mathcal{M}_{\beta\left(R, R^{\prime}\right)}^{\alpha}(x)=\int D U D_{\beta R}^{\alpha}[u(x)] U M_{R^{\prime}}(x) U^{-1}
$$

and

$$
\mathcal{M}_{\beta\left(R, R^{\prime}\right)}^{+\alpha}(x)=\int D U D_{\beta R}^{\alpha}\left[u^{+}(x)\right] U M_{R^{\prime}}(x) U^{-1},
$$

where $R^{\prime}$ is an arbitrary irreducible representation. $\forall V \in \mathcal{G}$ :

$$
\begin{aligned}
V \mathcal{M}_{\beta\left(R, R^{\prime}\right)}^{\alpha}(x) V^{-1} & =\int D U D_{\beta R}^{\alpha}[u(x)] V U M_{R^{\prime}}(x) U^{-1} V^{-1} \\
& =D_{\gamma R}^{\alpha}\left[v^{-1}(x)\right] \mathcal{M}_{\beta\left(R, R^{\prime}\right)}^{\gamma}(x)
\end{aligned}
$$

$$
\begin{aligned}
V \mathcal{M}_{\beta\left(R, R^{\prime}\right)}^{+\alpha}(x) V^{-1} & =\int D U D_{\beta R}^{\alpha}\left[u^{+}(x)\right] V U M_{R^{\prime}}(x) U^{-1} V^{-1} \\
& =\mathcal{M}_{\gamma\left(R, R^{\prime}\right)}^{+\alpha}(x) D_{\beta R}^{\gamma}[v(x)] .
\end{aligned}
$$

It seems that $\mathcal{M}_{\beta\left(R, R^{\prime}\right)}^{\alpha}(x)$ with the fixed $\beta$ transforms as a local operator in the representation $R$, while $\mathcal{M}_{\beta\left(R, R^{\prime}\right)}^{+\alpha}(x)$ with the fixed $\alpha$ transforms as a local operator in the representation $\bar{R}$.

However, in most cases, $\mathcal{M}_{\beta\left(R, R^{\prime}\right)}^{\alpha}(x)$ and $\mathcal{M}_{\beta\left(R, R^{\prime}\right)}^{+\alpha}(x)$ constructed in Eqs. (4.9) and (4.10) are actually 0. Since

$$
\begin{aligned}
\mathcal{M}_{\beta\left(R, R^{\prime}\right)}^{\alpha}(x)= & \int D U D_{\beta R}^{\alpha}[u(x) v(x)] U V M_{R^{\prime}}(x) V^{-1} U^{-1} \\
= & D_{\beta R}^{\gamma}[v(x)] \int D U D_{\gamma R}^{\alpha}[u(x)] \\
& \times U V M_{R^{\prime}}(x) V^{-1} U^{-1}
\end{aligned}
$$

for $V$ satisfying

$$
V M_{R^{\prime}}(x) V^{-1}=M_{R^{\prime}}(x),
$$

there will be

$$
\mathcal{M}_{\beta\left(R, R^{\prime}\right)}^{\alpha}(x)=\mathcal{M}_{\gamma\left(R, R^{\prime}\right)}^{\alpha}(x) D_{\beta R}^{\gamma}[v(x)]
$$

Here, $M_{R^{\prime}}$ and $\mathcal{M}_{\beta\left(R, R^{\prime}\right)}^{\alpha}$ share the same invariant group, which requires $D_{\beta R}^{\gamma}[v]=\delta_{\beta}^{\gamma}$, since otherwise, $\mathcal{M}_{\beta\left(R, R^{\prime}\right)}^{\alpha}=0$.

Let us consider the invariant group of $M_{R}(x)$. Suppose $G_{H}=\left\{e^{i H_{A} \alpha^{A}} \mid \forall \alpha^{A}\right\}$ is the Carton subgroup of $G$. The corresponding gauge transformation operators compose $\mathcal{G}_{H}$, which is a subgroup of $\mathcal{G} . \forall V \in \mathcal{G}_{H}$ with the related $v=e^{i H_{A} \alpha^{A}}$, for $M_{R}$ in Yang-Mills matter theory, from the gauge transformation rule in Eq. (4.5),

$$
V M_{R}(x) V^{-1}=M_{R}(x) .
$$

On the other hand, for $M_{R}$ in Chern-Simons matter theory, from the gauge transformation rule in Eq. (4.6),

$V M_{R}(x) V^{-1}=\exp \left\{-2 i k t r\left(H_{\vec{m}} H_{A}\right) \alpha^{A}(x)\right\} M_{R}(x)$.

In Yang-Mills matter theory, Eq. (4.14) requires $D_{\beta R}^{\gamma}[v]=\delta_{\beta}^{\gamma}, \forall v \in G_{H}$, which is possible only when $R$ is the identity representation $I$, in which case 


$$
\mathcal{M}_{(I, R)}(x)=\int D U U M_{R}(x) U^{-1}
$$

So, the only nonzero $\mathcal{M}$ that can be constructed is $\mathcal{M}_{(I, R)}(x)$, which is gauge invariant.

In Chern-Simons matter theory, $M_{R}$ transforms as in Eq. (4.15) under the action of $\mathcal{G}_{H}$. When $G=U(N)$, for $R^{\prime}$ labeled by

$$
H_{\vec{m}^{\prime}}=\operatorname{diag}\left(\frac{m_{1}^{\prime}}{2}, \ldots, \frac{m_{N}^{\prime}}{2}\right)
$$

considering $N U(1)$ transformation groups

$$
v_{i}=\operatorname{diag}(\underbrace{1, \ldots, 1}_{i-1}, e^{i \theta_{i}}, \underbrace{1, \ldots, 1}_{N-i}) \in G_{H}, \quad i=1, \ldots, N
$$

from Eq. (4.15), the corresponding $V_{i}$ will make

$$
V_{i} M_{R^{\prime}}(x) V_{i}^{-1}=\exp \left\{-i k m_{i}^{\prime} \theta_{i}(x)\right\} M_{R^{\prime}}(x) .
$$

Accordingly, for $\mathcal{M}_{\beta\left(R, R^{\prime}\right)}^{\alpha}(x) \neq 0, \quad \mathcal{M}_{\beta\left(R, R^{\prime}\right)}^{\alpha}(x)$ should satisfy

$\mathcal{M}_{\gamma\left(R, R^{\prime}\right)}^{\alpha}(x) D_{\beta R}^{\gamma}\left[v_{i}(x)\right]=\exp \left\{i k m_{i}^{\prime} \theta_{i}(x)\right\} \mathcal{M}_{\beta\left(R, R^{\prime}\right)}^{\alpha}(x)$.

$M_{R^{\prime}}$ and $\mathcal{M}_{\beta\left(R, R^{\prime}\right)}^{\alpha}$ must have the same invariant group and the opposite $U(1)$ charges, which is possible only when $R$ is the representation labeled by $k H_{\vec{m}^{\prime}}$ and $|\beta\rangle$ is the highestweight state in the representation $R$.

For example, when

$$
H_{\vec{m}^{\prime}}=\operatorname{diag}(\frac{m_{1}^{\prime}}{2}, \underbrace{0, \ldots, 0}_{N-1}),
$$

the invariant group of $M_{R^{\prime}}$ and $\mathcal{M}_{\beta\left(R, R^{\prime}\right)}^{\alpha}$ is $U(N-1)$, and $\mathcal{M}_{\beta\left(R, R^{\prime}\right)}^{\alpha}$ should satisfy

$\mathcal{M}_{\gamma\left(R, R^{\prime}\right)}^{\alpha}(x) D_{\beta R}^{\gamma}\left[v_{1}(x)\right]=\exp \left\{i k m_{1}^{\prime} \theta_{1}(x)\right\} \mathcal{M}_{\beta\left(R, R^{\prime}\right)}^{\alpha}(x)$.

$R$ must be the $k m_{1}^{\prime}$-symmetric representation, with $|\beta\rangle$ as the highest-weight state

$$
\underset{k m_{1}^{\prime}}{\otimes}(1, \underbrace{0, \ldots, 0}_{N-1}) .
$$

When

$$
H_{\vec{m}^{\prime}}=\operatorname{diag}(\frac{1}{2}, \frac{1}{2}, \underbrace{0, \ldots, 0}_{N-2}),
$$

the invariant group of $M_{R^{\prime}}$ and $\mathcal{M}_{\beta\left(R, R^{\prime}\right)}^{\alpha}$ is $S U(2) \times$ $U(N-2)$, and $\mathcal{M}_{\beta\left(R, R^{\prime}\right)}^{\alpha}$ should satisfy

$$
\mathcal{M}_{\gamma\left(R, R^{\prime}\right)}^{\alpha}(x) D_{\beta R}^{\gamma}\left[v_{i}(x)\right]=\exp \left\{i k \theta_{i}(x)\right\} \mathcal{M}_{\beta\left(R, R^{\prime}\right)}^{\alpha}(x), i=1,2 .
$$

$R$ must be an irreducible representation with the Young tableau containing $k$ boxes in the first two rows. $|\beta\rangle$ is the highest-weight state

$$
\begin{aligned}
\underset{k}{\otimes} & {[(1, \underbrace{0, \ldots, 0}_{N-1}) \otimes(0,1, \underbrace{0, \ldots, 0}_{N-2})} \\
- & (0,1, \underbrace{0, \ldots, 0}_{N-2}) \otimes(1, \underbrace{0, \ldots, 0}_{N-1})] .
\end{aligned}
$$

To conclude, in Chern-Simons matter theory with the level $k$, the monopole operator in representation $R$ labeled by $H_{\vec{m}}$ can be constructed as

$$
\mathcal{M}_{R}^{\alpha}(x)=\frac{1}{\sqrt{\mathcal{N}_{R}}} \int_{U \in \mathcal{G}} D U D_{1 R}^{\alpha}[u(x)] U M_{R / k}(x) U^{-1},
$$

where $|1\rangle$ is the highest-weight state in presentation $R$, and $M_{R / k}(x)$ is the monopole operator labeled by $H_{\vec{m} / k}$. Since $m_{i} / k$ must be integers, for $k>1$, not all of $R$ can be realized. For example, if $R$ is the fundamental representation, $k$ must be 1 ; if $R$ is the 2-symmetric representation, $k$ can only be 1 or 2 . The integration measure $D U$ is normalized with

$$
\int_{U \in \mathcal{G}} D U=1
$$

We also add a divided factor, $\sqrt{\mathcal{N}_{R}}$. Supposing that $\mathcal{G}_{S} \subset \mathcal{G}$ is the stationary group of the integrand, $\mathcal{N}_{R}$ is given by

$$
\mathcal{N}_{R}=\int_{U \in \mathcal{G}_{S}} D U
$$

By "stationary group," we mean $\forall V \in \mathcal{G}_{S}$,

$$
\begin{aligned}
& D_{1 R}^{\alpha}[u(x) v(x)] U V M_{R / k}(x) V^{-1} U^{-1} \\
& \quad=D_{1 R}^{\alpha}[u(x)] U M_{R / k}(x) U^{-1}, \quad \forall U \in \mathcal{G},
\end{aligned}
$$

which is equivalent to the condition

$$
D_{1 R}^{\beta}[v(x)] V M_{R / k}(x) V^{-1}=\delta_{1}^{\beta} M_{R / k}(x) .
$$

The associated $v$ could also compose a stationary group $G_{s}$, under which $|1\rangle$ is invariant up to a phase. $\forall v \in G_{s}$, $v|1\rangle=e^{i \theta}|1\rangle$, 


$$
\begin{aligned}
D_{1 R}^{\beta}[v(x)] & =\langle\beta|v(x)| 1\rangle=e^{i \theta(x)} \delta_{1}^{\beta}, \\
V M_{R / k}(x) V^{-1} & =e^{-i \theta(x)} M_{R / k} .
\end{aligned}
$$

Similarly, the monopole operator in the representation $\bar{R}$ is

$$
\mathcal{M}_{\alpha R}(x)=\frac{1}{\sqrt{\mathcal{N}_{R}}} \int_{U \in \mathcal{G}} D U D_{\alpha R}^{1}\left[u^{+}(x)\right] U M_{R / k}^{-1}(x) U^{-1},
$$

where $D_{\beta R}^{\alpha}\left(u^{+}\right)=D_{\alpha R}^{\beta *}(u)$ and $\mathcal{M}_{\alpha R}^{+}(x)=\mathcal{M}_{R}^{\alpha}(x)$. Under the local gauge transformation $V$,

$$
\begin{aligned}
V \mathcal{M}_{R}^{\alpha}(x) V^{-1} & =D_{\beta R}^{\alpha}\left[v^{-1}(x)\right] \mathcal{M}_{R}^{\beta}(x), \\
V \mathcal{M}_{\alpha R}(x) V^{-1} & =\mathcal{M}_{\beta R}(x) D_{\alpha R}^{\beta}[v(x)] .
\end{aligned}
$$

If $O_{R}^{\alpha}(x)$ and $O_{\alpha R}(x)$ are normal local operators in the representations $R$ and $\bar{R}$ with

$$
\begin{aligned}
V O_{R}^{\alpha}(x) V^{-1} & =D_{\beta R}^{\alpha}\left[v^{-1}(x)\right] O_{R}^{\beta}(x), \\
V O_{\alpha R}(x) V^{-1} & =O_{\beta R}(x) D_{\alpha R}^{\beta}[v(x)],
\end{aligned}
$$

then $\mathcal{M}_{R}^{\alpha}(x) O_{\alpha R}(x)$ and $\mathcal{M}_{\alpha R}(x) O_{R}^{\alpha}(x)$ will be gauge invariant. In this way, the vortex-charged gauge-invariant operators can be constructed.

Recall that in Eq. (2.4), $a_{i}$ can differ by a local gauge transformation. With $a_{i} \rightarrow a_{i}+\partial_{i} \sigma, \quad M_{R}(x) \rightarrow$ $U\left(H_{\vec{m}} \sigma\right) M_{R}(x)$ up to a phase. The different $a_{i}$ will also give rise to a different $\mathcal{M}_{R}$. Such ambiguity can be eliminated by Gauss constraint, which is imposed via a projection to the physical Hilbert space $\mathcal{H}_{\text {phy }}$ : $\forall|\psi\rangle \in \mathcal{H}_{\text {phy }}, \forall U \in \mathcal{G}, U|\psi\rangle=|\psi\rangle$. From Eqs. (4.27), (4.35), and (4.28), the action of the physical operator $\mathcal{M}_{R}^{\alpha}(x) O_{\alpha R}(x)$ on the physical state $\left|\psi_{1}\right\rangle$ is

$$
\begin{aligned}
\mathcal{M}_{R}^{\alpha}(x) O_{\alpha R}(x)\left|\psi_{1}\right\rangle= & \frac{1}{\sqrt{\mathcal{N}_{R}}} \int_{U \in \mathcal{G}} \operatorname{DUUM}_{R / k}(x) O_{1 R}(x)\left|\psi_{1}\right\rangle \\
= & \frac{1}{\sqrt{\mathcal{N}_{R}}} \int_{U \in \mathcal{G}} \operatorname{DUUU}\left(H_{\vec{m} / k} \sigma\right) \\
& \times M_{R / k}(x) O_{1 R}(x)\left|\psi_{1}\right\rangle \in \mathcal{H}_{\mathrm{phy}},
\end{aligned}
$$

and the projection of $\mathcal{M}_{R}^{\alpha}(x) O_{\alpha R}(x)$ in $\mathcal{H}_{\text {phy }}$ is

$$
\begin{gathered}
\left\langle\psi_{1}\left|\mathcal{M}_{R}^{\alpha}(x) O_{\alpha R}(x)\right| \psi_{2}\right\rangle=\frac{1}{\sqrt{\mathcal{N}_{R}}}\left\langle\psi_{1}\left|M_{R / k}(x) O_{1 R}(x)\right| \psi_{2}\right\rangle \\
=\frac{1}{\sqrt{\mathcal{N}_{R}}}\left\langle\psi_{1}\left|U\left(H_{\vec{m} / k} \sigma\right) M_{R / k}(x) O_{1 R}(x)\right| \psi_{2}\right\rangle
\end{gathered}
$$

So, with $a_{i}$ replaced by $a_{i}+\partial_{i} \sigma$, the physical state produced by $\mathcal{M}_{R}^{\alpha}(x) O_{\alpha R}(x)$ and the matrix elements of $\mathcal{M}_{R}^{\alpha}(x) O_{\alpha R}(x)$ in $\mathcal{H}_{\text {phy }}$ remain the same. We may select an arbitrary $a_{i}$ to construct $M_{R}$, and the obtained physical operators in $\mathcal{H}_{\text {phy }}$ are identical. In fact, when computing the matrix element in $\mathcal{H}_{\text {phy }}$, Eq. (4.37) can be equivalently interpreted in path-integral language. From Eq. (A7), the action of $M_{R / k}(x)$ produces a vortex-charged configuration, which is then combined with $O_{1 R}(x)$ to compensate the $U(1)$ charges. The integration over gauge-equivalent configurations gives the matrix element in physical Hilbert space.

In Chern-Simons matter theory with the gauge group $U(N)_{k} \times U(N)_{-k}, M_{\left[R_{1}, \bar{R}_{2}\right]}(x)$ is given by Eq. (3.16), and the corresponding monopole operator in the representation $\left(R_{1}, \bar{R}_{2}\right)$ can be constructed as

$$
\begin{aligned}
\mathcal{M}_{\hat{\alpha}\left(R_{1}, \bar{R}_{2}\right)}^{\alpha}(x)= & \frac{1}{\sqrt{\mathcal{N}_{R_{1}} \mathcal{N}_{R_{2}}}} \int_{U \in \mathcal{G}} D U \int_{\hat{U} \in \hat{\mathcal{G}}} D \hat{U} D_{1 R_{1}}^{\alpha}[u(x)] \\
& \times D_{\hat{\alpha} R_{2}}^{1}\left[\hat{u}^{+}(x)\right] U \hat{U} M_{\left[R_{1}, \bar{R}_{2}\right] / k}(x) U^{-1} \hat{U}^{-1} \\
= & \mathcal{M}_{R_{1}}^{\alpha}(x) \mathcal{M}_{\hat{\alpha} R_{2}}(x),
\end{aligned}
$$

where $\mathcal{M}_{R_{1}}^{\alpha}(x)$ and $\mathcal{M}_{\hat{\alpha} R_{2}}(x)$ are monopole operators for the first and second $U(N)$ groups. Under the local gauge transformation $V \hat{V}$,

$$
\begin{aligned}
& V \hat{V} \mathcal{M}_{\hat{\alpha}\left(R_{1}, \bar{R}_{2}\right)}^{\alpha}(x) V^{-1} \hat{V}^{-1} \\
& =D_{\beta R_{1}}^{\alpha}\left[v^{-1}(x)\right] \mathcal{M}_{\hat{\beta}\left(R_{1}, \bar{R}_{2}\right)}^{\beta}(x) D_{\hat{\alpha} R_{2}}^{\hat{\beta}}[\hat{v}(x)] .
\end{aligned}
$$

The topological charge $Q^{+}$is gauge invariant, so from Eqs. (3.18) and (4.39),

$$
\begin{aligned}
{\left[Q^{+}, \mathcal{M}_{\hat{\alpha}\left(R_{1}, \bar{R}_{2}\right)}^{\alpha}(x)\right] } & =-4 \operatorname{tr} H_{\vec{m}_{1} / k} \mathcal{M}_{\hat{\alpha}\left(R_{1}, \bar{R}_{2}\right)}^{\alpha}(x) \\
& =-4 t r H_{\vec{m}_{2} / k} \mathcal{M}_{\hat{\alpha}\left(R_{1}, \bar{R}_{2}\right)}^{\alpha}(x) .
\end{aligned}
$$

When $R_{1}=R_{2}=R, \quad \mathcal{M}_{\hat{\alpha}(R, \bar{R})}^{\alpha}(x)$ can be denoted as $\mathcal{M}_{\hat{\alpha} R}^{\alpha}(x)$.

$$
\mathcal{M}_{\hat{\alpha} R}^{\alpha}(x)=\mathcal{M}_{R}^{\alpha}(x) \mathcal{M}_{\hat{\alpha} R}(x)
$$

is in the $(R, \bar{R})$ representation of $U(N) \times U(N)$, and $\mathcal{M}_{\hat{\alpha} R}^{\alpha+}(x)=\mathcal{M}_{\alpha R}^{\hat{\alpha}}(x)$.

In $\mathrm{ABJM}$ theory, monopole operators with $R_{1}=R_{2}$ are used to construct gauge-invariant chiral operators. The generic monopole operators with $R_{1} \neq R_{2}$ should also exist and are required to make the large- $N$ spectrum of the protected operators agree with that of the gravity states [22]. 


\section{THE CONTRACTION RELATIONS FOR MONOPOLE OPERATORS}

In this section, we will prove some contraction relations for the monopole operators $\mathcal{M}_{R}^{\alpha}(x)$ and $\mathcal{M}_{\alpha R}(x)$ that will be used in Sec. X.

Consider the Chern-Simons matter theory with the gauge group $U(N)$ and the level $k=1$. If $R$ is the fundamental representation $\mathbf{N}$, the related $H_{\vec{m}}$ is

$$
H_{[1]}=\operatorname{diag}(\frac{1}{2}, \underbrace{0, \ldots, 0}_{N-1}) .
$$

The corresponding monopole operator is

$$
\mathcal{M}^{a}(x)=\frac{1}{\sqrt{\mathcal{N}_{[1]}}} \int_{U \in \mathcal{G}} D U D_{1}^{a}[u(x)] U M_{[1]}(x) U^{-1},
$$

where $a=1, \ldots, N, D_{b}^{a}[u]$ is the fundamental representation of $u \in U(N)$, and $M_{[1]}(x)$ is $M_{R}(x)$, with $H_{\vec{m}}$ given by Eq. (5.1).

$$
\mathcal{M}_{a}(x)=\frac{1}{\sqrt{\mathcal{N}_{[1]}}} \int_{U \in \mathcal{G}} D U D_{a}^{1}\left[u^{+}(x)\right] U M_{[1]}^{-1}(x) U^{-1}
$$

is the monopole operator in the antifundamental representation $\overline{\mathbf{N}}$, and $\mathcal{M}_{a}^{+}(x)=\mathcal{M}^{a}(x)$. Under the local gauge transformation,

$$
\begin{aligned}
V \mathcal{M}^{a}(x) V^{-1} & =D_{b}^{a}\left[v^{-1}(x)\right] \mathcal{M}^{b}(x), \\
V \mathcal{M}_{a}(x) V^{-1} & =\mathcal{M}_{b}(x) D_{a}^{b}[v(x)] .
\end{aligned}
$$

The contraction of $\mathcal{M}_{a}(x)$ and $\mathcal{M}^{a}(x)$ is

$$
\begin{aligned}
\mathcal{M}_{a}(x) \mathcal{M}^{a}(x) & =\frac{1}{\mathcal{N}_{[1]}} \int_{U, V \in \mathcal{G}} D_{V D U D_{a}^{1}\left[v^{+}(x)\right] D_{1}^{a}[u(x)] V M_{[1]}^{-1}(x) V^{-1} U M_{[1]}(x) U^{-1}} \\
& =\frac{1}{\mathcal{N}_{[1]}} \int_{U \in \mathcal{G}} D U D_{1}^{1}[u(x)] \int_{V \in \mathcal{G}} D V V\left[M_{[1]}^{-1}(x) U M_{[1]}(x) U^{-1}\right] V^{-1}
\end{aligned}
$$

$\forall W \in \mathcal{G}$,

$$
\begin{aligned}
& \int_{V \in \mathcal{G}} D V V\left[M_{[1]}^{-1}(x) U M_{[1]}(x) U^{-1}\right] V^{-1} \\
& =\int_{V \in \mathcal{G}} D V V W\left[M_{[1]}^{-1}(x) U M_{[1]}(x) U^{-1}\right] W^{-1} V^{-1} .
\end{aligned}
$$

For the given $U$, one can always find some $W$ with

$W\left[M_{[1]}^{-1}(x) U M_{[1]}(x) U^{-1}\right] W^{-1}=e^{i \theta}\left[M_{[1]}^{-1}(x) U M_{[1]}(x) U^{-1}\right]$.

As a result, Eq. (5.6) $\neq 0$ only when $U \in \mathcal{G}_{S}, u \in G_{s}=$ $U(1) \times U(N-1)$, and then

$$
\begin{gathered}
D_{1}^{1}[u(x)] U M_{[1]}(x) U^{-1}=M_{[1]}(x), \\
\mathcal{M}_{a}(x) \mathcal{M}^{a}(x)=\frac{1}{\mathcal{N}_{[1]}} \int_{U \in \mathcal{G}_{S}} D U \int_{V \in \mathcal{G}} D V=1 .
\end{gathered}
$$

$\mathcal{M}^{a}$ and $\mathcal{M}_{a}$ in the representations $\mathbf{N}$ and $\overline{\mathbf{N}}$ do not exist when $k>1$.

When $k=1$, the 2-symmetric representation $\mathbf{N}_{\mathrm{sym}}^{2}$ is labeled by

$$
H_{[2]}=\operatorname{diag}(1, \underbrace{0, \ldots, 0}_{N-1}) \text {. }
$$

The corresponding monopole operator is

$$
\mathcal{M}^{a b}(x)=\frac{1}{\sqrt{\mathcal{N}_{[2]}}} \int_{U \in \mathcal{G}} D U D_{1}^{a}[u(x)] D_{1}^{b}[u(x)] U M_{[2]}(x) U^{-1},
$$

where $M_{[2]}(x)$ is $M_{R}(x)$, with $R$ labeled by Eq. (5.10). The monopole operator in the $\overline{\mathbf{N}}_{\text {sym }}^{2}$ representation is

$$
\begin{aligned}
\mathcal{M}_{a b}(x)= & \frac{1}{\sqrt{\mathcal{N}_{[2]}}} \int_{U \in \mathcal{G}} D U D_{a}^{1}\left[u^{+}(x)\right] D_{b}^{1}\left[u^{+}(x)\right] \\
& \times U M_{[2]}^{-1}(x) U^{-1}
\end{aligned}
$$

and $\mathcal{M}_{a b}^{+}(x)=\mathcal{M}^{a b}(x)$. Under the local gauge transformation,

$$
\begin{aligned}
V \mathcal{M}^{a b}(x) V^{-1} & =D_{c}^{a}\left[v^{-1}(x)\right] D_{d}^{b}\left[v^{-1}(x)\right] \mathcal{M}^{c d}(x), \\
V \mathcal{M}_{a b}(x) V^{-1} & =\mathcal{M}_{c d}(x) D_{a}^{c}[v(x)] D_{b}^{d}[v(x)]
\end{aligned}
$$

The contraction of $\mathcal{M}_{a b}(x)$ and $\mathcal{M}^{b c}(x)$ is 


$$
\begin{aligned}
\mathcal{M}_{a b}(x) \mathcal{M}^{b c}(x) & =\frac{1}{\mathcal{N}_{[2]}} \int_{V, U \in \mathcal{G}} D V D U D_{a}^{1}\left[v^{+}(x)\right] D_{b}^{1}\left[v^{+}(x)\right] D_{1}^{b}[u(x)] D_{1}^{c}[u(x)] V M_{[2]}^{-1}(x) V^{-1} U M_{[2]}(x) U^{-1} \\
& =\frac{1}{\mathcal{N}_{[2]}} \int_{U \in \mathcal{G}} D U D_{1}^{1}[u(x)] D_{1}^{b}[u(x)] \int_{V \in \mathcal{G}} D V D_{a}^{1}\left[v^{+}(x)\right] D_{b}^{c}[v(x)] V\left[M_{[2]}^{-1}(x) U M_{[2]}(x) U^{-1}\right] V^{-1} .
\end{aligned}
$$

$\forall W \in \mathcal{G}$,

$$
\begin{aligned}
& \int_{V \in \mathcal{G}} D V D_{a}^{1}\left[v^{+}(x)\right] D_{b}^{c}[v(x)] V\left[M_{[2]}^{-1}(x) U M_{[2]}(x) U^{-1}\right] V^{-1} \\
& \quad=\int_{V \in \mathcal{G}} D V D_{a}^{1}\left[w^{+}(x) v^{+}(x)\right] D_{b}^{c}[v(x) w(x)] V W\left[M_{[2]}^{-1}(x) U M_{[2]}(x) U^{-1}\right] W^{-1} V^{-1} .
\end{aligned}
$$

For the given $U$, one can always find some $W$ with

$$
W\left[M_{[2]}^{-1} U M_{[2]} U^{-1}\right] W^{-1}=e^{i \theta}\left[M_{[2]}^{-1} U M_{[2]} U^{-1}\right] .
$$

Equation $(5.15) \neq 0$ only when

$$
D_{a}^{1}\left[w^{+} v^{+}\right] D_{b}^{c}[v w]=e^{-i \theta} D_{a}^{1}\left[v^{+}\right] D_{b}^{c}[v], \quad \forall v \in G .
$$

We should at least have

$$
u H_{[2]} u^{+}=\operatorname{diag}(\underbrace{0, \ldots, 0}_{m}, 1, \underbrace{0, \ldots, 0}_{N-m-1}) .
$$

Moreover, $m$ must be 0 , since otherwise, $D_{1}^{1}[u]=0$. So, we have $U \in \mathcal{G}_{s}, u H_{[2]} u^{+}=H_{[2]}$, in which case $D_{1}^{b}[u] \neq 0$ only when $b=1$, and $\left(D_{1}^{1}[u(x)]\right)^{2} U M_{[2]}(x) U^{-1}=M_{[2]}$.

$$
\begin{aligned}
\mathcal{M}_{a b}(x) \mathcal{M}^{b c}(x)= & \frac{1}{\mathcal{N}_{[2]}} \int_{U \in \mathcal{G}_{S}} D U \int_{V \in \mathcal{G}} D V D_{a}^{1}\left[v^{+}(x)\right] \\
& \times D_{1}^{c}[v(x)] \\
= & \int_{v \in G} d v D_{a}^{1}\left[v^{+}(x)\right] D_{1}^{c}[v(x)] \\
= & \frac{\delta_{a}^{c}}{N}
\end{aligned}
$$

where we have used

$$
\int_{u \in G} d u D_{\beta R}^{\alpha}\left[u^{+}\right] D_{\sigma R}^{\rho}[u]=\frac{1}{d_{R}} \delta_{\beta}^{\rho} \delta_{\sigma}^{\alpha},
$$

and $\int_{u \in G} d u=1$. Again, $\mathcal{M}_{a b}(x) \mathcal{M}^{b a}(x)=1$.

The same conclusion holds for $k=2$ with $H_{[2]}$ in Eq. (5.10) replaced by $H_{[1]}$ in Eq. (5.1). When $k>2$, $\mathcal{M}^{a b}(x)$ and $\mathcal{M}_{a b}(x)$ in the representations $\mathbf{N}_{\text {sym }}^{2}$ and $\overline{\mathbf{N}}_{\text {sym }}^{2}$ do not exist. When $k=1$, with $H_{\vec{m}}$ taken to be

$$
H_{[1,1]}=\operatorname{diag}(\frac{1}{2}, \frac{1}{2}, \underbrace{0, \ldots, 0}_{N-2}) \text {, }
$$

we can also get $\mathcal{M}^{\prime a b}(x)$ in the 2-antisymmetric representation $\mathbf{N}_{\text {asym }}^{2}$ and $\mathcal{M}_{a b}^{\prime}(x)$ in the conjugate representation $\overline{\mathbf{N}}_{\text {asym }}^{2}$.

In Chern-Simons matter theory with the gauge group $U(N)_{1} \times U(N)_{-1}$, the monopole operator in the representation $(\mathbf{N}, \overline{\mathbf{N}})$ is

$$
\begin{aligned}
\mathcal{M}_{\hat{a}}^{a}(x)= & \frac{1}{N_{[1]}} \int D U D \hat{U} D_{1}^{a}[u(x)] \\
& \times D_{\hat{a}}^{1}\left[\hat{u}^{+}(x)\right] U \hat{U} M_{[1, \overline{1}]}(x) U^{-1} \hat{U}^{-1} \\
= & \mathcal{M}^{a}(x) \mathcal{M}_{\hat{a}}(x),
\end{aligned}
$$

where $M_{[1, \overline{1}]}(x)$ is given by Eq. (3.16) with $k=1$ and $H_{\vec{m}}=H_{[1]}, a, \hat{a}=1, \ldots, N . \mathcal{M}_{\hat{a}}^{a+}(x)=\mathcal{M}_{a}^{\hat{a}}(x)$. Under the local gauge transformation,

$V \hat{V} \mathcal{M}_{\hat{a}}^{a}(x) V^{-1} \hat{V}^{-1}=D_{b}^{a}\left[v^{-1}(x)\right] \mathcal{M}_{\hat{b}}^{b}(x) D_{\hat{a}}^{\hat{b}}[\hat{v}(x)]$.

The monopole operators in the $\left(\mathbf{N}_{\text {sym }}^{2}, \overline{\mathbf{N}}_{\text {sym }}^{2}\right)$ and $\left(\overline{\mathbf{N}}_{\text {sym }}^{2}, \mathbf{N}_{\text {sym }}^{2}\right)$ representations are

$\mathcal{M}_{\hat{a} \hat{b}}^{a b}(x)=\mathcal{M}^{a b}(x) \mathcal{M}_{\hat{a} \hat{b}}(x), \mathcal{M}_{a b}^{\hat{a} \hat{b}}(x)=\mathcal{M}_{a b}(x) \mathcal{M}^{\hat{a} \hat{b}}(x)$.

Here, $\mathcal{M}_{\hat{a} \hat{b}}^{a b+}(x)=\mathcal{M}_{a b}^{\hat{a} \hat{b}}(x)$. From Eq. (5.19),

$$
\begin{aligned}
\mathcal{M}_{\hat{a} \hat{b}}^{a b}(x) \mathcal{M}_{a c}^{\hat{a} \hat{c}}(x) & =\mathcal{M}^{a b}(x) \mathcal{M}_{\hat{a} \hat{b}}(x) \mathcal{M}_{a c}(x) \mathcal{M}^{\hat{a} \hat{c}}(x) \\
& =\frac{1}{N^{2}} \delta_{c}^{b} \delta_{\hat{b}}^{\hat{c}} .
\end{aligned}
$$

With a rescaling

$\mathcal{M}_{\hat{a} \hat{b}}^{a b}(x) \rightarrow N \mathcal{M}_{\hat{a} \hat{b}}^{a b}(x), \quad \mathcal{M}_{a c}^{\hat{a} \hat{c}}(x) \rightarrow N \mathcal{M}_{a c}^{\hat{a} \hat{c}}(x)$,

we get 


$$
\mathcal{M}_{\hat{a} \hat{b}}^{a b}(x) \mathcal{M}_{a c}^{\hat{a} \hat{c}}(x)=\delta_{c}^{b} \delta_{\hat{b}}^{\hat{c}}
$$

which is the contraction relation needed in Sec. X.

When $k=1$, except for $\mathcal{M}_{\hat{a} \hat{b}}^{a b}(x)$ and $\mathcal{M}_{a b}^{\hat{a} \hat{b}}(x)$, there are six additional monopole operators carrying the vortex charge \pm 4 :

$$
\begin{aligned}
\mathcal{M}_{\hat{a} \hat{b}}^{\prime a b}(x) & =\mathcal{M}^{\prime a b}(x) \mathcal{M}_{\hat{a} \hat{b}}^{\prime}(x), \\
\mathcal{M}_{\hat{a} \hat{b}}^{\prime a \hat{b}}(x) & =\mathcal{M}_{a b}^{\prime}(x) \mathcal{M}^{\prime a \hat{b}}(x)
\end{aligned}
$$

in the $\left(\mathbf{N}_{\text {asym }}^{2}, \overline{\mathbf{N}}_{\text {asym }}^{2}\right)$ and $\left(\overline{\mathbf{N}}_{\text {asym }}^{2}, \mathbf{N}_{\text {asym }}^{2}\right)$ representations;

$$
\begin{aligned}
& \mathcal{M}_{\hat{a} \hat{b}}^{\prime \prime a b}(x)=\mathcal{M}^{a b}(x) \mathcal{M}_{\hat{a} \hat{b}}^{\prime}(x), \\
& \mathcal{M}_{a b}^{\prime \prime \hat{b} \hat{b}}(x)=\mathcal{M}_{a b}(x) \mathcal{M}^{\prime \hat{a} \hat{b}}(x)
\end{aligned}
$$

in the $\left(\mathbf{N}_{\text {sym }}^{2}, \overline{\mathbf{N}}_{\text {asym }}^{2}\right)$ and $\left(\overline{\mathbf{N}}_{\text {sym }}^{2}, \mathbf{N}_{\text {asym }}^{2}\right)$ representations; and

$$
\begin{aligned}
& \mathcal{M}_{\hat{a} \hat{b}}^{\prime \prime \prime a b}(x)=\mathcal{M}^{\prime a b}(x) \mathcal{M}_{\hat{a} \hat{b}}(x), \\
& \mathcal{M}_{\hat{a} \hat{b}}^{\prime \prime \prime \hat{b}}(x)=\mathcal{M}_{a b}^{\prime}(x) \mathcal{M}^{\hat{a} \hat{b}}(x)
\end{aligned}
$$

in the $\left(\mathbf{N}_{\text {asym }}^{2}, \overline{\mathbf{N}}_{\text {sym }}^{2}\right)$ and $\left(\overline{\mathbf{N}}_{\text {asym }}^{2}, \mathbf{N}_{\text {sym }}^{2}\right)$ representations. As will be discussed in Sec. X, in ABJM theory, such operators can be used to construct the superconformal current multiplets.

\section{CLASSICAL CONFORMAL DIMENSION OF MONOPOLE OPERATORS}

In this section, we will compute the classical conformal dimension of the monopole operator from the action of the dilation $D$. Unlike in Ref. [2], the calculation is entirely carried out at the kinetical level without the knowledge of the exact Lagrangian. The classical conformal dimension may receive quantum corrections from the interaction unless there are supersymmetries to protect it.
In $(d+1)$-dimensional conformal field theory, for a field $\phi$ with the conformal dimension $\Delta$, the conjugate momentum $\pi$ should have the conformal dimension $\Delta^{\prime}=d-\Delta$. The action of the dilatation operator $D$ is

$-i[D, \phi]=\Delta \phi+x^{\mu} \partial_{\mu} \phi, \quad-i[D, \pi]=(d-\Delta) \pi+x^{\mu} \partial_{\mu} \pi$,

where $\mu=0,1, \ldots, d$. The charge density $\rho$ has the conformal dimension $d$ with

$$
-i[D, \rho]=d \rho+x^{\mu} \partial_{\mu} \rho .
$$

We will only consider the 3D Chern-Simons matter theory, which can be classically conformal invariant. The gauge field $A$ and its conjugate momentum both have the conformal dimension 1, in consistence with the commutation relation [Eq. (3.4)]. The charge density $\rho$ has the conformal dimension 2. At $x^{0}=0$,

$$
-i\left[D, A_{i}\right]=A_{i}+x^{j} \partial_{j} A_{i}, \quad-i[D, \rho]=2 \rho+x^{i} \partial_{i} \rho,
$$

where $i, j=1,2$. The monopole operator $M_{R}(x)$ is given by Eq. (3.6). Let $M_{R}(x)=\exp \left\{\operatorname{im}_{R}(x)\right\}$, where

$$
\begin{aligned}
m_{R}(x)= & \frac{k}{2 \pi} \int d^{2} y \operatorname{tr} H_{\vec{m}}\left\{\epsilon^{i j} A_{j}(y) a_{i}(x, y)\right. \\
& \left.+\left[\frac{i}{2} \epsilon^{i j}\left[A_{i}(y), A_{j}(y)\right]+\frac{2 \pi}{k} \rho(y)\right] \omega(x, y)\right\} .
\end{aligned}
$$

$a_{i}(x, y)$ is taken to be

$$
a_{i}(x, y)=2 \epsilon_{j i} \frac{x^{j}-y^{j}}{|x-y|^{2}} .
$$

From Eqs. (6.3)-(6.5),

$$
\begin{aligned}
-i\left[D, m_{R}(x)\right]= & \frac{k}{2 \pi} \int d^{2} y \operatorname{tr} H_{\vec{m}}\left\{\epsilon^{i j}\left[A_{j}(y)+y^{k} \partial_{k}^{y} A_{j}(y)\right] a_{i}(x, y)+2\left[\frac{i}{2} \epsilon^{i j}\left[A_{i}(y), A_{j}(y)\right]+\frac{2 \pi}{k} \rho(y)\right] \omega(x, y)\right. \\
& \left.+y^{k} \partial_{k}^{y}\left[\frac{i}{2} \epsilon^{i j}\left[A_{i}(y), A_{j}(y)\right]+\frac{2 \pi}{k} \rho(y)\right] \omega(x, y)\right\} \\
= & x^{k} \partial_{k} m_{R}(x),
\end{aligned}
$$

where we have used

$$
-i\left[D, M_{R}(x)\right]=x^{i} \partial_{i} M_{R}(x)
$$

$$
\begin{aligned}
\left(x^{j}-y^{j}\right) \partial_{j}^{y} a_{i} & =a_{i}, \quad\left(x^{i}-y^{i}\right) a_{i}=0, \\
\partial_{k}^{y} a_{i}(x, y) & =-\partial_{k}^{x} a_{i}(x, y), \quad \partial_{k}^{y} \omega(x, y)=-\partial_{k}^{x} \omega(x, y) .
\end{aligned}
$$

Therefore,
The classical conformal dimension of $M_{R}(x)$ is 0. Equation (6.8) applies for $M_{R}(x)$ with $x^{0}=0$; however, since $D=$ $e^{i H x^{0}} D e^{-i H x^{0}}-H x^{0}$ and $M_{R}\left(x^{i}, x^{0}\right)=e^{i H x^{0}} M_{R}\left(x^{i}, 0\right) e^{-i H x^{0}}$, from Eq. (6.8), 


$$
-i\left[D, M_{R}(x)\right]=x^{\mu} \partial_{\mu} M_{R}(x)
$$

can be recovered.

$M_{R}(x)$ is a special kind of gauge transformation. In 3D Chern-Simons matter theory, consider the generic local gauge transformation operator

$$
U(\alpha)=\exp \left\{-i \int d^{2} y \operatorname{tr}[\alpha(y) \Lambda(y)]\right\}
$$

with $\Lambda$ given by Eq. (3.5). At $x^{0}=0,-i[D, \Lambda]=$ $2 \Lambda+x^{i} \partial_{i} \Lambda$. Under the scaling transformation $e^{-i \lambda D}$,

$$
\begin{aligned}
& e^{-i \lambda D} \Lambda(x) e^{i \lambda D}=e^{2 \lambda} \Lambda\left(e^{\lambda} x\right) \\
e^{-i \lambda D} U(\alpha) e^{i \lambda D} & =\exp \left\{-i \int d^{2} y \operatorname{tr}\left[e^{2 \lambda} \alpha(y) \Lambda\left(e^{\lambda} y\right)\right]\right\} \\
& =\exp \left\{-i \int d^{2} y \operatorname{tr}\left[\alpha\left(e^{-\lambda} y\right) \Lambda(y)\right]\right\} \\
& =U\left(\alpha^{\prime}\right)
\end{aligned}
$$

where $\alpha^{\prime}$ is the gauge transformation parameter with $\alpha^{\prime}(y)=\alpha\left(e^{-\lambda} y\right)$. In $M_{R}(x), \omega$ is scale invariant with $\omega\left(e^{\lambda} x, y\right)=\omega\left(x, e^{-\lambda} y\right)$, so

$$
e^{-i \lambda D} M_{R}(x) e^{i \lambda D}=M_{R}\left(e^{\lambda} x\right)
$$

from which Eq. (6.8), and then Eq. (6.9), is obtained again.

As for the scaling transformation of $\mathcal{M}_{R}(x)$, from Eq. (4.27),

$$
\begin{aligned}
e^{-i \lambda D} \mathcal{M}_{R}^{\alpha}(x) e^{i \lambda D}= & \frac{1}{\sqrt{\mathcal{N}_{R}}} \int_{U \in \mathcal{G}} D U D_{1 R}^{\alpha}\left[u^{\prime}\left(e^{\lambda} x\right)\right] \\
& \times U^{\prime} M_{R / k}\left(e^{\lambda} x\right) U^{\prime-1} \\
= & \mathcal{M}_{R}^{\alpha}\left(e^{\lambda} x\right),
\end{aligned}
$$

where for $U$ with the transformation matrix $u, U^{\prime}=$ $e^{-i \lambda D} U e^{i \lambda D}$ is the gauge transformation with the transformation matrix $u^{\prime}: u^{\prime}(x)=u\left(e^{-\lambda} x\right), D U=D U^{\prime}$. The classical conformal dimension of $\mathcal{M}_{R}^{\alpha}(x)$, and similarly, those of $\mathcal{M}_{\alpha R}(x)$ and $\mathcal{M}_{\hat{\alpha}\left(R_{1}, \bar{R}_{2}\right)}^{\alpha}(x)$, are all 0 .

Note that, for $\mathcal{M}_{R}$ to have the conformal dimension 0 under the action of $D$, we have selected a particular $a_{i}$ in Eq. (6.5), although the generic $a_{i}$ is given by Eq. (2.4). $D$ is not gauge invariant. The gauge-invariant version of $D$ is $\mathcal{D}$, which, when acting on physical operators, reduces to $D$. $\mathcal{M}_{R}$ 's constructed from the gauge equivalent $a_{i}$ will all have the conformal dimension 0 under the action of $\mathcal{D}$. We will return to this point in Sec. IX.

\section{SUPERSYMMETRY TRANSFORMATION OF MONOPOLE OPERATORS}

In this section, we will study the supersymmetry transformation of the monopole operator in the 3D super-Yang-Mills (SYM) theory and the ABJM theory. Concretely, we will calculate the action of the singular gauge transformation $M_{R}$ on supercharges, from which the commutator of the supercharges and $M_{R}$ can be obtained.

The ordinary local gauge transformation operator $U(\alpha)$ is characterized by the Lie-algebra-valued function $\alpha$, which is well defined everywhere. With $\alpha$ replaced by the multivalued function $H_{\vec{m}} \omega$, we get $U\left(H_{\vec{m}} \omega\right)=M_{R}$. The gauge-invariant operators are obviously invariant under the action of $U(\alpha)$. For example, for the supercharge $\mathcal{Q}$, we have $U(\alpha) \mathcal{Q} U^{-1}(\alpha)=\mathcal{Q}$. However, not all gauge-invariant operators would be invariant under the action of $U\left(H_{\vec{m}} \omega\right)$. In the following, we will compute the singular gauge transformation of the supercharges in the SYM theory and the ABJM theory explicitly.

Consider the 3D SYM theory with the gauge group $U(N)$, where the fundamental fields are $\left(A_{i}, \Phi^{I}, \Psi\right)$ with the conjugate momentum $\left(\Pi^{i}, \Pi_{I}, \Pi\right)$, $i=1,2, I=1, \ldots, 7$. Supercharges are gauge-invariant operators composed by $D_{i} \Phi^{I},\left[\Phi^{I}, \Phi^{J}\right], \Pi^{i}, \Pi_{I}, \Pi, \Psi$, and $F$, among which $F$ transforms as

$$
\begin{aligned}
M_{R}(x) F(y) M_{R}^{-1}(x)= & e^{i H_{\vec{m}} \omega(x, y)} F(y) e^{-i H_{\vec{m}} \omega(x, y)} \\
& +8 \pi H_{\vec{m}} \delta^{2}(x-y)
\end{aligned}
$$

under the action of $M_{R}(x)$, while the other operators all transform as

$$
M_{R}(x) D_{i} \Phi^{I}(y) M_{R}^{-1}(x)=e^{i H_{\vec{m}} \omega(x, y)} D_{i} \Phi^{I}(y) e^{-i H_{\vec{m}} \omega(x, y)} .
$$

As a result, for

$$
\begin{aligned}
\mathcal{Q}= & \int d^{2} y \operatorname{tr}\left[\left(\frac{1}{4} F \epsilon_{i j} \Gamma^{i j}-\frac{i}{2}\left[\Phi_{I}, \Phi_{J}\right] \Gamma^{I J}+\Pi_{i} \Gamma^{0 i}\right.\right. \\
& \left.\left.+\Pi_{I} \Gamma^{0 I}+D_{i} \Phi_{J} \Gamma^{i J}\right) \Gamma^{0} \Psi\right],
\end{aligned}
$$

where $\Gamma$ 's are $32 \times 32$ gamma matrices in $10 \mathrm{D}$ spacetime, we have

$$
M_{R}(x) \mathcal{Q} M_{R}^{-1}(x)=\mathcal{Q}+2 \pi \epsilon_{i j} \Gamma^{i j} \Gamma^{0} \operatorname{tr}\left[H_{\vec{m}} \Psi(x)\right]
$$

and

$$
\left[\mathcal{Q}, M_{R}(x)\right]=-2 \pi \epsilon_{i j} \Gamma^{i j} \Gamma^{0} \operatorname{tr}\left[H_{\vec{m}} \Psi(x)\right] M_{R}(x) .
$$


In fact, for Yang-Mills matter theory, $M_{R}(x)$ given by Eq. (3.3) takes the same form as the ordinary local gauge transformation operator with $a_{i}=\partial_{i} \omega$ satisfied as well. The obstruction for $M_{R}(x)$ to be a genuine gauge transformation is $\epsilon^{i j} \partial_{i} a_{j}(x, y)=4 \pi \delta^{2}(x-y) \neq 0$, so when computing the supersymmetry variation of $M_{R}(x)$, we only need to consider terms producing $\epsilon^{i j} \partial_{i} a_{j}$. The supersymmetry variation of $\Pi^{i}$ is

$$
\left[\mathcal{Q}, \Pi^{i}\right]=-i\left[\Gamma^{j i} \Gamma^{0} \partial_{j} \Psi+\cdots\right]
$$

where "..." are terms with no derivatives, so

$$
\begin{aligned}
{\left[\mathcal{Q}, M_{R}(x)\right] } & =\int d^{2} y \operatorname{tr} H_{\vec{m}}\left(\Gamma^{j i} \Gamma^{0} \partial_{j} \Psi a_{i}\right) M_{R}(x) \\
& =\int d^{2} y \operatorname{tr} H_{\vec{m}}\left(-\Gamma^{j i} \Gamma^{0} \Psi \partial_{j} a_{i}\right) M_{R}(x) \\
& =-2 \pi \epsilon_{i j} \Gamma^{i j} \Gamma^{0} \operatorname{tr}\left[H_{\vec{m}} \Psi(x)\right] M_{R}(x)
\end{aligned}
$$

is obtained again.

It is possible to construct the scalar-dressed monopole operator preserving part of the super-symmetry. Since

$$
\left[\mathcal{Q}, \Phi^{I}\right]=-i \Gamma^{I} \Psi
$$

for the operator $\exp \left\{-4 \pi \operatorname{tr}\left[H_{\vec{m}} \Phi^{I}(x)\right]\right\} M_{R}(x)$, the supersymmetry variation is

$$
\left[\bar{\epsilon} \mathcal{Q}, \exp \left\{-4 \pi t r\left[H_{\vec{m}} \Phi^{I}(x)\right]\right\} M_{R}(x)\right]=-4 \pi \bar{\epsilon}\left(-i \Gamma^{I}+\Gamma^{1} \Gamma^{2} \Gamma^{0}\right) \operatorname{tr}\left[H_{\vec{m}} \Psi(x)\right] \exp \left\{-4 \pi t r\left[H_{\vec{m}} \Phi^{I}(x)\right]\right\} M_{R}(x)
$$

Equation (7.8) $=0$ for $\epsilon$ satisfying $\bar{\epsilon} \Gamma^{1} \Gamma^{2} \Gamma^{0} \Gamma^{I}=i \bar{\epsilon}$, so $\exp \left\{-4 \pi \operatorname{tr}\left[H_{\vec{m}} \Phi^{I}(x)\right]\right\} M_{R}(x) \quad$ is $\quad$ a $\quad 1 / 2 \quad$ BPS operator.

Next, consider the $U(N)_{k} \times U(N)_{-k}$ ABJM theory with $\mathcal{N}=6$ supersymmetry. Following the convention in Ref. [34], the field content consists of complex scalars $X_{A \hat{a}}^{a}$, spinors $\Psi_{\hat{a}}^{A a}$, and their adjoints $X_{a}^{A \hat{a}}, \Psi_{A a}^{\hat{a}}$, transforming as $(N, \bar{N})$ and $(\bar{N}, N)$ representations of the gauge group. $A=1, \ldots, 4$, and $a, \hat{a}=1, \ldots, N$. The $U(N)$ gauge fields are Hermitian matrices $A_{b}^{a}$ and $\hat{A}_{\hat{b}}^{\hat{a}}$. The covariant derivatives are

$$
\begin{aligned}
& D_{\mu} X_{A}=\partial_{\mu} X_{A}-i\left(A_{\mu} X_{A}-X_{A} \hat{A}_{\mu}\right), \\
& D_{\mu} X^{A}=\partial_{\mu} X^{A}-i\left(\hat{A}_{\mu} X^{A}-X^{A} A_{\mu}\right),
\end{aligned}
$$

with similar formulas for the spinors, and $\mu=0,1,2$. The action of the ABJM theory is [34]

$$
S=S_{\mathrm{kin}}+S_{S C}+S_{F}+S_{S},
$$

with the kinetic term

$$
S_{\mathrm{kin}}=\frac{k}{2 \pi} \int d^{3} x \operatorname{tr}\left(-D^{\mu} X^{A} D_{\mu} X_{A}+i \bar{\Psi}_{A} \gamma^{\mu} D_{\mu} \Psi^{A}\right),
$$

the Chern-Simons term

$$
\begin{aligned}
S_{S C}= & \frac{k}{2 \pi} \int d^{3} x \epsilon^{\mu \nu \lambda} \operatorname{tr}\left(\frac{1}{2} A_{\mu} \partial_{\nu} A_{\lambda}\right. \\
& \left.-\frac{i}{3} A_{\mu} A_{\nu} A_{\lambda}-\frac{1}{2} \hat{A}_{\mu} \partial_{\nu} \hat{A}_{\lambda}+\frac{i}{3} \hat{A}_{\mu} \hat{A}_{\nu} \hat{A}_{\lambda}\right),
\end{aligned}
$$

the fermionic interaction

$$
\begin{aligned}
S_{F}= & i \int d^{3} x \epsilon^{A B C D} \operatorname{tr}\left(\bar{\Psi}_{A} X_{B} \Psi_{C} X_{D}\right)-\epsilon_{A B C D} \operatorname{tr}\left(\bar{\Psi}^{A} X^{B} \Psi^{C} X^{D}\right) \\
& +\operatorname{tr}\left(\bar{\Psi}^{A} \Psi_{A} X_{B} X^{B}-\bar{\Psi}_{A} \Psi^{A} X^{B} X_{B}\right. \\
& \left.+2 \bar{\Psi}_{A} \Psi^{B} X^{A} X_{B}-2 \bar{\Psi}^{B} \Psi_{A} X_{B} X^{A}\right),
\end{aligned}
$$

and the scalar potential

$$
\begin{aligned}
S_{S}= & \frac{1}{3} \int d^{3} x \operatorname{tr}\left(X^{A} X_{A} X^{B} X_{B} X^{C} X_{C}+X_{A} X^{A} X_{B} X^{B} X_{C} X^{C}\right. \\
& \left.+4 X_{A} X^{B} X_{C} X^{A} X_{B} X^{C}-6 X^{A} X_{B} X^{B} X_{A} X^{C} X_{C}\right) .
\end{aligned}
$$

Here, $\gamma^{\mu}$ are $2 \times 2$ Dirac matrices satisfying $\left\{\gamma^{\mu}, \gamma^{\nu}\right\}=$ $2 \eta^{\mu \nu} \cdot \bar{\Psi}^{A}$ and $\bar{\Psi}_{A}$ are transpositions of $\Psi^{A}$ and $\Psi_{A}$ multiplied by $\gamma^{0}$.

Under the $\mathcal{N}=6$ supersymmetry transformation [34],

$$
\begin{aligned}
\delta X_{A} & =i \Gamma_{A B}^{I} \bar{\Psi}^{B} \varepsilon^{I}, \\
\delta \Psi_{A} & =\Gamma_{A B}^{I} \gamma^{\mu} \varepsilon^{I} D_{\mu} X^{B}+N_{A}^{I} \varepsilon^{I}, \\
\delta A_{\mu} & =-\Gamma_{A B}^{I} \bar{\Psi}^{A} \gamma_{\mu} \varepsilon^{I} X^{B}-\tilde{\Gamma}^{I A B} X_{B} \bar{\Psi}_{A} \gamma_{\mu} \varepsilon^{I}, \\
\delta \hat{A}_{\mu} & =-\Gamma_{A B}^{I} X^{B} \bar{\Psi}^{A} \gamma_{\mu} \varepsilon^{I}-\tilde{\Gamma}^{I A B} \bar{\Psi}_{A} \gamma_{\mu} \varepsilon^{I} X_{B},
\end{aligned}
$$

where

$N_{A}^{I}=\Gamma_{A B}^{I}\left(X^{C} X_{C} X^{B}-X^{B} X_{C} X^{C}\right)-2 \Gamma_{B C}^{I} X^{B} X_{A} X^{C}$.

$\Gamma^{I}$, with $I=1, \ldots, 6$, are $4 \times 4$ matrices satisfying $\Gamma_{A B}^{I}=-\Gamma_{B A}^{I}$.

$$
\tilde{\Gamma}^{I A B}=\frac{1}{2} \epsilon^{A B C D} \Gamma_{C D}^{I}=-\left(\Gamma_{A B}^{I}\right)^{*},
$$




$$
\Gamma^{I} \tilde{\Gamma}^{J}+\Gamma^{J} \tilde{\Gamma}^{I}=2 \delta^{I J}
$$

The six supercharges are given by [34]

$$
\begin{aligned}
\mathcal{Q}^{I}= & -\frac{k}{2 \pi} \int d^{2} x \operatorname{tr}\left[\left(-\Gamma_{A B}^{I} \gamma^{\nu} D_{\nu} X^{B}+N_{A}^{I}\right) \gamma_{0} \Psi^{A}\right] \\
& +\operatorname{tr}\left[\left(\tilde{\Gamma}^{I A B} \gamma^{\nu} D_{\nu} X_{B}+N^{I A}\right) \gamma_{0} \Psi_{A}\right]
\end{aligned}
$$

$N^{I A}=\left(N_{A}^{I}\right)^{+}$. The six conformal supercharges are given by [34]

$$
\begin{aligned}
\mathcal{S}^{I}= & -\frac{k}{2 \pi} \int d^{2} x \operatorname{tr}\left[\gamma^{\mu} x_{\mu}\left(-\Gamma_{A B}^{I} \gamma^{\nu} D_{\nu} X^{B}+N_{A}^{I}\right) \gamma_{0} \Psi^{A}\right] \\
& +\operatorname{tr}\left[\gamma^{\mu} x_{\mu}\left(\tilde{\Gamma}^{I A B} \gamma^{\nu} D_{\nu} X_{B}+N^{I A}\right) \gamma_{0} \Psi_{A}\right] \\
& -\Gamma_{A B}^{I} \operatorname{tr}\left(X^{B} \gamma_{0} \Psi^{A}\right)+\tilde{\Gamma}^{I A B} \operatorname{tr}\left(X_{B} \gamma_{0} \Psi_{A}\right) .
\end{aligned}
$$

In parallel with the discussion for 3D SYM theory, $\mathcal{Q}^{I}$ and $\mathcal{S}^{I}$ are gauge invariant and do not contain $F$; thus, they are invariant under the action of $M_{R}(x)$.

$$
\begin{array}{ccc}
M_{R}(x) \mathcal{Q}^{I} M_{R}^{-1}(x) & =\mathcal{Q}^{I}, & {\left[\mathcal{Q}^{I}, M_{R}(x)\right]=0,} \\
M_{R}(x) \mathcal{S}^{I} M_{R}^{-1}(x) & =\mathcal{S}^{I}, & {\left[\mathcal{S}^{I}, M_{R}(x)\right]=0 .}
\end{array}
$$

$M_{R}(x)$ is SUSY invariant.

In ABJM theory, $M_{R}(x)$ is given by Eq. (3.16), in which the terms involving $a_{i}$ are $\epsilon^{i j} A_{j} a_{i}$ and $\epsilon^{i j} \hat{A}_{j} a_{i}$, so to compute the supersymmetry variation of $M_{R}(x)$, we only need to consider $\delta A$ and $\delta \hat{A}$. However, in Eqs. (7.17) and (7.18), $\delta A$ and $\delta \hat{A}$ do not contain derivatives, so in $\left[\mathcal{Q}^{I}, M_{R}(x)\right]$ and $\left[\mathcal{S}^{I}, M_{R}(x)\right], \epsilon^{i j} \partial_{i} a_{j}$ cannot be produced. The difference between $M_{R}(x)$ and $U(\alpha)$ does not have a manifestation here, so just like $U(\alpha), M_{R}(x)$ commutes with the supercharge.

Finally, for $\mathcal{M}_{\hat{\alpha}\left(R_{1}, \bar{R}_{2}\right)}^{\alpha}(x)$ in the representation $\left(R_{1}, \bar{R}_{2}\right)$ constructed in Eq. (4.39), $\left[\mathcal{Q}^{I}, M_{\left[R_{1}, \bar{R}_{2}\right]}(x)\right]=0$, $\left[\mathcal{S}^{I}, M_{\left[R_{1}, \bar{R}_{2}\right]}(x)\right]=0, \quad\left[\mathcal{Q}^{I}, U\right]=\left[\mathcal{Q}^{I}, \hat{U}\right]=0, \quad$ and $\left[\mathcal{S}^{I}, U\right]=\left[\mathcal{S}^{I}, \hat{U}\right]=0$, so

$$
\left[\mathcal{Q}^{I}, \mathcal{M}_{\hat{\alpha}\left(R_{1}, \bar{R}_{2}\right)}^{\alpha}(x)\right]=0, \quad\left[\mathcal{S}^{I}, \mathcal{M}_{\hat{\alpha}\left(R_{1}, \bar{R}_{2}\right)}^{\alpha}(x)\right]=0 .
$$

\section{DERIVATIVE AND COVARIANT DERIVATIVE OF MONOPOLE OPERATORS}

In this section, we will compute the derivative and the covariant derivative of the monopole operators $M_{R}(x)$ and $\mathcal{M}_{R}(x)$ in 3D Yang-Mills matter theory and Chern-Simons matter theory. We find that in Chern-Simons matter theory, $M_{R}(x)$ and $\mathcal{M}_{R}(x)$ are covariantly constant.

The monopole operator $M_{R}(x)$ at the point $x$ is characterized by the function $H_{\vec{m}} a_{i}(x, y)$. Under the infinitesimal translation $x \rightarrow x+\xi, \quad M_{R}(x) \rightarrow M_{R}(x+\xi)$, $a_{i}(x, y) \rightarrow a_{i}(x+\xi, y)=a_{i}(x, y-\xi)$.
In 3D Yang-Mills matter theory, $M_{R}(x)$ is given by

$$
\begin{aligned}
M_{R}(x)= & \exp \left\{i \int d ^ { 2 } y \operatorname { t r } H _ { \vec { m } } \left[\Pi^{i}(y) a_{i}(x, y)\right.\right. \\
& \left.\left.+i\left[A_{i}(y), \Pi^{i}(y)\right] \omega(x, y)+\rho(y) \omega(x, y)\right]\right\} .
\end{aligned}
$$

From Eq. (6.7), the derivative of $M_{R}(x)$ is

$$
\begin{aligned}
\partial_{i} M_{R}(x)= & -i \int d^{2} y \operatorname{tr} H_{\vec{m}}\left[\Pi^{j} \partial_{i} a_{j}\right. \\
& \left.+i\left[A_{j}, \Pi^{j}\right] \partial_{i} \omega+\rho \partial_{i} \omega\right] M_{R}(x) \\
= & \left\{4 \pi i \epsilon_{j i} \operatorname{tr}\left[H_{\vec{m}} \Pi^{j}(x)\right]\right. \\
& \left.+i \int d^{2} y \operatorname{tr}\left[H_{\vec{m}} a_{i}(x, y) \Lambda(y)\right]\right\} M_{R}(x),
\end{aligned}
$$

where $\Lambda=\partial_{i} \Pi^{i}-i\left[A_{i}, \Pi^{i}\right]-\rho$. We can also calculate the covariant derivative of $M_{R}(x)$. Consider the local gauge transformation operator $U(\alpha)=\exp \{-i G(\alpha)\}$ with the generator

$$
G(\alpha)=\int d^{2} y \operatorname{tr}[\alpha(y) \Lambda(y)]
$$

Under the action of $M_{R}(x), G\left(A_{i}\right)$ transforms as

$$
\begin{aligned}
M_{R}(x) G\left(A_{i}\right) M_{R}^{-1}(x) & =G\left(A_{i}\right)+\int d^{2} y \operatorname{tr}\left[H_{\vec{m}} a_{i}(x, y) \Lambda(y)\right] \\
& =G\left(A_{i}\right)+G\left(H_{\vec{m}} a_{i}\right)
\end{aligned}
$$

so

$$
\left[G\left(A_{i}\right), M_{R}(x)\right]=-G\left(H_{\vec{m}} a_{i}\right) M_{R}(x) .
$$

The covariant derivative of $M_{R}(x)$ is

$$
\begin{aligned}
D_{i} M_{R}(x) & =\partial_{i} M_{R}(x)+i\left[G\left(A_{i}\right), M_{R}(x)\right] \\
& =4 \pi i \epsilon_{j i} \operatorname{tr}\left[H_{\vec{m}} \Pi^{j}(x)\right] M_{R}(x) .
\end{aligned}
$$

Compared with Eq. (8.2), the right-hand side of Eq. (8.6) only contains local operators.

Of course, the derivative could be directly obtained from the action of the momentum operator $P_{i}$. For example, in a 3D $U(N)$ Yang-Mills matter theory with the matter part composed of a scalar $\phi$ and a spinor $\psi$, both in adjoint representation of $U(N)$,

$$
P_{i}=\int d^{2} y \operatorname{tr}\left(\Pi_{\phi} \partial_{i} \phi+\Pi_{\psi} \partial_{i} \psi+\Pi^{j} \partial_{i} A_{j}\right),
$$

where $\Pi_{\phi}$ and $\Pi_{\psi}$ are conjugate momenta of $\phi$ and $\psi$. For $f=\left(\Pi_{\phi}, \phi, \Pi_{\psi}, \psi, \Pi^{i}, A_{i}\right), \partial_{i} f(x)=i\left[P_{i}, f(x)\right]$. Under the action of $M_{R}(x)$, 
$M_{R}(x) P_{i} M_{R}^{-1}(x)=P_{i}-G\left(H_{\vec{m}} a_{i}\right)-4 \pi \epsilon_{j i} t r\left[H_{\vec{m}} \Pi^{j}(x)\right]$,

so

$$
\begin{aligned}
\partial_{i} M_{R}(x) & =i\left[P_{i}, M_{R}(x)\right] \\
& =\left\{4 \pi i \epsilon_{j i} t r\left[H_{\vec{m}} \Pi^{j}(x)\right]+i G\left(H_{\vec{m}} a_{i}\right)\right\} M_{R}(x),
\end{aligned}
$$

which is consistent with Eq. (8.2).

The gauge-invariant completion of $P_{i}$ is

$$
\begin{aligned}
\mathcal{P}_{i} & =\int d^{2} y \operatorname{tr}\left(\Pi_{\phi} D_{i} \phi+\Pi_{\psi} D_{i} \psi+\Pi^{j} F_{i j}\right) \\
& =P_{i}+\int d^{2} y \operatorname{tr}\left(A_{i} \Lambda\right) \\
& =P_{i}+G\left(A_{i}\right) .
\end{aligned}
$$

When $f=\left(\Pi_{\phi}, \phi, \Pi_{\psi}, \psi\right),\left[\mathcal{P}_{i}, f(x)\right]=-i D_{i} f(x)$. For the gauge field $A_{j}$,

$$
\left[\mathcal{P}_{i}, A_{j}(x)\right]=-i F_{i j}(x)
$$

From Eqs. (8.5), (8.9), and (8.10), Eq. (8.6) is recovered.

In 3D Chern-Simons matter theory with the level $k$, the monopole operator $M_{R}(x)$ is

$$
\begin{aligned}
M_{R}(x)= & \exp \left\{\frac { i k } { 2 \pi } \int d ^ { 2 } y \operatorname { t r } H _ { \vec { m } } \left[\epsilon^{i j} A_{j}(y) a_{i}(x, y)\right.\right. \\
& \left.\left.+\frac{i}{2} \epsilon^{i j}\left[A_{i}(y), A_{j}(y)\right] \omega(x, y)+\frac{2 \pi}{k} \rho(y) \omega(x, y)\right]\right\} .
\end{aligned}
$$

Still, consider a Chern-Simons matter theory with the gauge group $U(N)$ and the field content $\left(A_{i}, \phi, \psi\right)$. The momentum operator is

$$
P_{i}=\int d^{2} y \operatorname{tr}\left(\Pi_{\phi} \partial_{i} \phi+\Pi_{\psi} \partial_{i} \psi-\frac{k}{4 \pi} A_{i} F\right) .
$$

For $f=\left(\Pi_{\phi}, \phi, \Pi_{\psi}, \psi, A_{i}\right), \partial_{i} f(x)=i\left[P_{i}, f(x)\right]$. Under the action of $M_{R}(x)$,

$$
\begin{aligned}
M_{R}(x) P_{i} M_{R}^{-1}(x)= & P_{i}-\int d^{2} y \operatorname{tr}\left[H_{\vec{m}} a_{i}(x, y) \Lambda(y)\right] \\
& -2 k \operatorname{tr}\left[H_{\vec{m}} A_{i}(x)+H_{\vec{m}} H_{\vec{m}} a_{i}(x, x)\right],
\end{aligned}
$$

where

$$
\Lambda=\frac{k}{4 \pi} F-i\left(\left[\phi, \Pi_{\phi}\right]-\left\{\psi, \Pi_{\psi}\right\}\right)
$$

So

$$
\begin{aligned}
\partial_{i} M_{R}(x)= & i\left[P_{i}, M_{R}(x)\right] \\
= & i G\left(H_{\vec{m}} a_{i}\right) M_{R}(x)+2 k i t r\left[H_{\vec{m}} A_{i}(x)\right. \\
& \left.+H_{\vec{m}} H_{\vec{m}} a_{i}(x, x)\right] M_{R}(x) .
\end{aligned}
$$

The gauge-invariant momentum operator is

$$
\begin{aligned}
\mathcal{P}_{i} & =\int d^{2} y \operatorname{tr}\left(\Pi_{\phi} D_{i} \phi+\Pi_{\psi} D_{i} \psi\right) \\
& =P_{i}+\int d^{2} y \operatorname{tr}\left(A_{i} \Lambda\right) \\
& =P_{i}+G\left(A_{i}\right)
\end{aligned}
$$

Especially in pure CS theory, $\mathcal{P}_{i}=0$, and moreover, with $A_{0}=0, H=0$ [33]. When $f=\left(\Pi_{\phi}, \phi, \Pi_{\psi}, \psi\right)$, $\left[\mathcal{P}_{i}, f(x)\right]=-i D_{i} f(x)$. For the gauge field $A_{j}$,

$$
\left[\mathcal{P}_{i}, A_{j}\right]=\frac{2 \pi}{k} \epsilon_{i j}\left(\left[\phi, \Pi_{\phi}\right]-\left\{\psi, \Pi_{\psi}\right\}\right)
$$

in contrast to Eq. (8.11). $\mathcal{P}_{i}$ is gauge invariant and does not contain $F$, so

$M_{R}(x) \mathcal{P}_{i} M_{R}^{-1}(x)=\mathcal{P}_{i}, \quad D_{i} M_{R}(x)=i\left[\mathcal{P}_{i}, M_{R}(x)\right]=0$.

$M_{R}(x)$ is covariantly constant. On the other hand, in YangMills matter theory, $M_{R}(x)$ is not covariantly constant, merely because $\mathcal{P}_{i}$ given by Eq. (8.10) has $F$ involved.

Next, let us compute the derivative and the covariant derivative of the monopole operator $\mathcal{M}_{R}(x)$ in representation $R$.

$$
\mathcal{M}_{R}^{\alpha}(x)=\frac{1}{\sqrt{N_{R}}} \int D U D_{1 R}^{\alpha}[u(x)] U M_{R / k}(x) U^{-1} .
$$

Aside from $M_{R / k}(x), D_{1 R}^{\alpha}[u(x)]$ also has explicit $x$ dependence, so we should check whether $\partial_{i} \mathcal{M}_{R}(x)$ can still be realized by the action of $P_{i}$.

The action of the translation operator $\exp \left\{i P_{i} \xi^{i}\right\}$ transforms $M_{R}(x)$ into

$$
M_{R}(x+\xi)=\exp \left\{i P_{i} \xi^{i}\right\} M_{R}(x) \exp \left\{-i P_{i} \xi^{i}\right\} .
$$

For $\mathcal{M}_{R}^{\alpha}(x)$, we have 


$$
\begin{aligned}
& \exp \left\{i P_{i} \xi^{i}\right\} \mathcal{M}_{R}^{\alpha}(x) \exp \left\{-i P_{i} \xi^{i}\right\} \\
& =\frac{1}{\sqrt{N_{R}}} \int D U^{\prime} D_{1 R}^{\alpha}\left[u^{\prime}(x+\xi)\right] U^{\prime} M_{R / k}(x+\xi) U^{\prime-1} \\
& =\mathcal{M}_{R}^{\alpha}(x+\xi)
\end{aligned}
$$

where

$$
U^{\prime}=\exp \left\{i P_{i} \xi^{i}\right\} U \exp \left\{-i P_{i} \xi^{i}\right\}
$$

is the gauge transformation with the related transformation matrix $u^{\prime}(x)=u(x-\xi), D U=D U^{\prime}$. So,

$$
\partial_{i} \mathcal{M}_{R}^{\alpha}(x)=i\left[P_{i}, \mathcal{M}_{R}^{\alpha}(x)\right]
$$

is still valid.

The covariant derivative operator $\mathcal{P}_{i}$ is gauge invariant, $\left[U, \mathcal{P}_{i}\right]=0$, so

$$
\begin{aligned}
D_{i} \mathcal{M}_{R}^{\alpha}(x) & =i\left[\mathcal{P}_{i}, \mathcal{M}_{R}^{\alpha}(x)\right] \\
& =\frac{i}{\sqrt{N_{R}}} \int D U D_{1 R}^{\alpha}[u(x)] U\left[\mathcal{P}_{i}, M_{R / k}(x)\right] U^{-1}=0 .
\end{aligned}
$$

$\mathcal{M}_{R}^{\alpha}(x)$ is also covariantly constant.

Since $\mathcal{M}_{R}^{\alpha}(x)$ is an operator in the representation $R$, $D_{i} \mathcal{M}_{R}^{\alpha}(x)$ can be written explicitly. Consider the gaugeinvariant operator $O_{\alpha R}(x) \mathcal{M}_{R}^{\alpha}(x)$, with $O_{\alpha R}(x)$ in representation $\bar{R}$ constructed from the matter fields.

$$
D_{i} O_{\alpha R}(x)=i\left[\mathcal{P}_{i}, O_{\alpha R}(x)\right]=\partial_{i} O_{\alpha R}(x)+i O_{\beta R}(x) A_{\alpha i}^{\beta}(x) .
$$

When acting on the gauge-invariant operators, $\mathcal{P}_{i}$ reduces to $P_{i}$, so

$$
\begin{aligned}
\partial_{i}\left[O_{\alpha R}(x) \mathcal{M}_{R}^{\alpha}(x)\right] & =i\left[\mathcal{P}_{i}, O_{\alpha R}(x)\right] \mathcal{M}_{R}^{\alpha}(x)+i O_{\alpha R}(x)\left[\mathcal{P}_{i}, \mathcal{M}_{R}^{\alpha}(x)\right] \\
& =\left[\partial_{i} O_{\alpha R}(x)+i O_{\beta R}(x) A_{\alpha i}^{\beta}(x)\right] \mathcal{M}_{R}^{\alpha}(x)+i O_{\alpha R}(x)\left[\mathcal{P}_{i}, \mathcal{M}_{R}^{\alpha}(x)\right]
\end{aligned}
$$

The covariant derivative of $\mathcal{M}_{R}^{\alpha}(x)$ is

$$
\begin{aligned}
D_{i} \mathcal{M}_{R}^{\alpha}(x) & =i\left[\mathcal{P}_{i}, \mathcal{M}_{R}^{\alpha}(x)\right] \\
& =\partial_{i} \mathcal{M}_{R}^{\alpha}(x)-i A_{\beta i}^{\alpha}(x) \mathcal{M}_{R}^{\beta}(x)=0 .
\end{aligned}
$$

For the 3D Chern-Simons matter theory with the gauge group $U(N)_{k} \times U(N)_{-k}, \mathcal{M}_{\hat{\alpha} R}^{\alpha}(x)$ in the representation $(R, \bar{R})$ is covariantly constant with

$$
\begin{aligned}
D_{i} \mathcal{M}_{\hat{\alpha} R}^{\alpha}(x)= & \partial_{i} \mathcal{M}_{\hat{\alpha} R}^{\alpha}(x)-i A_{\beta i}^{\alpha}(x) \mathcal{M}_{\hat{\alpha} R}^{\beta}(x) \\
& +i \mathcal{M}_{\hat{\beta} R}^{\alpha}(x) \hat{A}_{\hat{\alpha} i}^{\hat{\beta}}(x)=0
\end{aligned}
$$

We should also consider the derivative and the covariant derivative along the time direction. Since $A_{0}=\hat{A}_{0}=0$ and $D_{0}=\partial_{0}$,

$$
D_{0} M_{R}(x)=\partial_{0} M_{R}(x)=i\left[H, M_{R}(x)\right],
$$

where $H$ is the Hamiltonian. In Chern-Simons matter theory, $H$ is gauge invariant and does not contain $F$; thus, it would commute with $M_{R}(x) . D_{0} M_{R}(x)=\partial_{0} M_{R}(x)=0$. $[U, H]=[\hat{U}, H]=0$, so $D_{0} \mathcal{M}_{R}^{\alpha}(x)=\partial_{0} \mathcal{M}_{R}^{\alpha}(x)=0$ and $D_{0} \mathcal{M}_{\hat{\alpha} R}^{\alpha}(x)=\partial_{0} \mathcal{M}_{\hat{\alpha} R}^{\alpha}(x)=0$.

Finally, in the above discussion, we take the particular Yang-Mills matter theory and the Chern-Simons matter theory as the examples, but the result is independent of the matter content. We may conclude that in the generic ChernSimons matter theories, the monopole operators are covariantly constant.

\section{THE CONSERVATION OF THE $S O(8)$ R-SYMMETRY CURRENT IN ABJM THEORY}

ABJM theory has the manifest $S U(4) \times U(1)_{J}$ global symmetry with $S U(4)$ and $U(1)_{J}$ realized by 15 traceless currents [35]

$$
\begin{aligned}
j_{\mu B}^{A}= & -\frac{i k}{2 \pi}\left\{\left[X_{a}^{A \hat{a}}\left(D_{\mu} X_{B}\right)_{\hat{a}}^{a}-\left(D_{\mu} X^{A}\right)_{a}^{\hat{a}} X_{B \hat{a}}^{a}+i \bar{\Psi}_{\hat{a}}^{A a} \gamma_{\mu} \Psi_{B a}^{\hat{a}}\right]\right. \\
& \left.-\frac{1}{4} \delta_{B}^{A}\left[X_{a}^{C \hat{a}}\left(D_{\mu} X_{C}\right)_{\hat{a}}^{a}-\left(D_{\mu} X^{C}\right)_{a}^{\hat{a}} X_{C \hat{a}}^{a}+i \bar{\Psi}_{\hat{a}}^{C a} \gamma_{\mu} \Psi_{C a}^{\hat{a}}\right]\right\}
\end{aligned}
$$

and a single current

$$
j_{\mu}=\frac{1}{4 \pi} \epsilon_{\mu \nu \lambda}\left(t r F^{\nu \lambda}+\operatorname{tr} \hat{F}^{\nu \lambda}\right)
$$

whose conservation follows from the equations of motion and the Bianchi identity. Using the Gauss constraint, Eq. (9.2) can also be written as the global $U(1)$ charge current.

When $k=1,2$, with the help of the monopole operator, 12 extra currents $[28,35]$

$$
\begin{aligned}
j_{\mu}^{A B}= & -\frac{i k}{4 \pi} \mathcal{M}_{\hat{a} \hat{b}}^{a b}\left[X_{a}^{A \hat{a}}\left(D_{\mu} X^{B}\right)_{b}^{\hat{b}}-\left(D_{\mu} X^{A}\right)_{a}^{\hat{a}} X_{b}^{B \hat{b}}\right. \\
& \left.+\frac{i}{2} \epsilon^{A B C D} \bar{\Psi}_{C a}^{\hat{a}} \gamma_{\mu} \Psi_{D b}^{\hat{b}}\right]
\end{aligned}
$$


and their adjoints $j_{\mu A B}=j_{\mu}^{A B+}$ can be constructed, which, if are conserved, will offer the off-diagonal charges, making the original $S U(4) \times U(1)_{J}$ symmetry enhanced to $S O(8)$.
Let us consider the current conservation equation for $j_{\mu}^{A B}$. In Appendix $B$, with the equations of motion plugged in, $\partial^{\mu} j_{\mu}^{A B}$ could be written as

$$
\begin{aligned}
\partial^{\mu} j_{\mu}^{A B}= & -\frac{i k}{4 \pi}\left(D^{\mu} \mathcal{M}\right)_{\hat{a} \hat{b}}^{a b}\left[X_{a}^{A \hat{a}}\left(D_{\mu} X^{B}\right)_{b}^{\hat{b}}-\left(D_{\mu} X^{A}\right)_{a}^{\hat{a}} X_{b}^{B \hat{b}}+\frac{i}{2} \epsilon^{A B C D} \bar{\Psi}_{C a}^{\hat{a}} \gamma_{\mu} \Psi_{D b}^{\hat{b}}\right] \\
& -\left(W_{C}^{D} \mathcal{M}\right)_{\hat{a} \hat{b}}^{a b}\left[X_{a}^{B \hat{a}}\left(W_{D}^{C} X^{A}\right)_{b}^{\hat{b}}-X_{a}^{A \hat{a}}\left(W_{D}^{C} X^{B}\right)_{b}^{\hat{b}}\right]-\frac{1}{2}\left(W_{C}^{C} \mathcal{M}\right)_{\hat{a} \hat{b}}^{a b}\left[X_{a}^{A \hat{a}}\left(W_{D}^{D} X^{B}\right)_{b}^{\hat{b}}-X_{a}^{B \hat{a}}\left(W_{D}^{D} X^{A}\right)_{b}^{\hat{b}}\right] \\
& -\frac{i}{2} \bar{\Psi}_{C b}^{\hat{b}}\left(V_{\mathrm{DE}} \mathcal{M}\right)_{\hat{a} \hat{b}}^{a b}\left(\epsilon^{A B C D} X_{a}^{E \hat{a}}-2 \epsilon^{A B C E} X_{a}^{D \hat{a}}\right) \\
& -\frac{i}{2}\left[\left(U^{B C} \mathcal{M}\right)_{\hat{a} \hat{b}}^{a b} X_{a}^{A \hat{a}}-\left(U^{A C} \mathcal{M}\right)_{\hat{a} \hat{b}}^{a b} X_{a}^{B \hat{a}}+2\left(U^{C A} \mathcal{M}\right)_{\hat{a} \hat{b}}^{a b} X_{a}^{B \hat{a}}-2\left(U^{C B} \mathcal{M}\right)_{\hat{a} \hat{b}}^{a b} X_{a}^{A \hat{a}}\right] \Psi_{C b}^{\hat{b}} .
\end{aligned}
$$

$D^{\mu} \mathcal{M}$ is the covariant derivative of $\mathcal{M}$ :

$$
\begin{aligned}
\left(D^{\mu} \mathcal{M}\right)_{\hat{a} \hat{b}}^{a b}= & \partial^{\mu} \mathcal{M}_{\hat{a} \hat{b}}^{a b}-i A_{c}^{\mu a} \mathcal{M}_{\hat{a} \hat{b}}^{c b}-i A_{c}^{\mu b} \mathcal{M}_{\hat{a} \hat{b}}^{a c} \\
& +i \mathcal{M}_{\hat{c} \hat{b}}^{a b} \hat{A}_{\hat{a}}^{\mu \hat{c}}+i \mathcal{M}_{\hat{a} \hat{c}}^{a b} \hat{A}_{\hat{b}}^{\mu \hat{c}}
\end{aligned}
$$

$W_{D}^{C}$ is a $U(N) \times U(N)$ gauge variation whose actions on $X^{B}$ and $\mathcal{M}$ are given by

$$
\left(W_{D}^{C} X^{B}\right)_{a}^{\hat{a}} \equiv\left(X^{C} X_{D}\right)_{\hat{b}}^{\hat{a}} X_{a}^{B \hat{b}}-X_{b}^{B \hat{a}}\left(X_{D} X^{C}\right)_{a}^{b}
$$

and

$$
\begin{aligned}
\left(W_{D}^{C} \mathcal{M}\right)_{\hat{a} \hat{b}}^{a b} \equiv & \left(X_{D} X^{C}\right)_{c}^{a} \mathcal{M}_{\hat{a} \hat{b}}^{c b}+\left(X_{D} X^{C}\right)_{c}^{b} \mathcal{M}_{\hat{a} \hat{b}}^{a c} \\
& -\mathcal{M}_{\hat{c} \hat{b}}^{a b}\left(X^{C} X_{D}\right)_{\hat{a}}^{\hat{c}}-\mathcal{M}_{\hat{a} \hat{c}}^{a b}\left(X^{C} X_{D}\right)_{\hat{b}}^{\hat{c}},
\end{aligned}
$$

respectively. $V_{C D}$ and $U^{C B}$ are $U(N) \times U(N)$ gauge variations whose actions on $\mathcal{M}$ are given by

$$
\begin{aligned}
\left(V_{C D} \mathcal{M}\right)_{\hat{a} \hat{b}}^{a b} \equiv & \left(X_{D} \Psi_{C}\right)_{c}^{a} \mathcal{M}_{\hat{a} \hat{b}}^{c b}+\left(X_{D} \Psi_{C}\right)_{c}^{b} \mathcal{M}_{\hat{a} \hat{b}}^{a c} \\
& -\mathcal{M}_{\hat{c} \hat{b}}^{a b}\left(\Psi_{C} X_{D}\right)_{\hat{a}}^{\hat{c}}-\mathcal{M}_{\hat{a} \hat{c}}^{a b}\left(\Psi_{C} X_{D}\right)_{\hat{b}}^{\hat{c}}
\end{aligned}
$$

and

$$
\begin{aligned}
\left(U^{C B} \mathcal{M}\right)_{\hat{a} \hat{b}}^{a b} \equiv & \left(\bar{\Psi}^{B} X^{C}\right)_{c}^{a} \mathcal{M}_{\hat{a} \hat{b}}^{c b}+\left(\bar{\Psi}^{B} X^{C}\right)_{c}^{b} \mathcal{M}_{\hat{a} \hat{b}}^{a c} \\
& -\mathcal{M}_{\hat{c} \hat{b}}^{a b}\left(X^{C} \bar{\Psi}^{B}\right)_{\hat{a}}^{\hat{c}}-\mathcal{M}_{\hat{a} \hat{c}}^{a b}\left(X^{C} \bar{\Psi}^{B}\right)_{\hat{b}}^{\hat{c}},
\end{aligned}
$$

respectively. The current conservation equation $\partial^{\mu} j_{\mu}^{A B}=0$ requires

$D^{\mu} \mathcal{M}=0, \quad W_{D}^{C} \mathcal{M}=0, \quad V_{C D} \mathcal{M}=0, \quad U^{C D} \mathcal{M}=0$,

$\forall C, D=1, \ldots, 4$. Equation (9.10) is obtained from the equations of motion and the algebraic calculations, with no explicit form for monopole operators assumed. In the following, we will show that the constructed monopole operators satisfy Eq. (9.10).

In Sec. VIII, $D^{\mu} \mathcal{M}=0$ has been proven. $V_{C D} \mathcal{M}=0$ and $U^{C D} \mathcal{M}=0$ are related to $W_{D}^{C} \mathcal{M}=0$ by a $\mathcal{N}=6$ supersymmetry transformation. Supposing that $\Gamma_{A B}^{I} \varepsilon^{I}=\eta_{A B}$, Eqs. (7.15)-(7.18) could be written as

$$
\begin{aligned}
\delta X_{A}= & i \bar{\Psi}^{B} \eta_{A B}, \\
\delta \Psi_{A}= & \gamma^{\mu} \eta_{A B} D_{\mu} X^{B}+\eta_{A B}\left(X^{C} X_{C} X^{B}-X^{B} X_{C} X^{C}\right) \\
& -2 \eta_{B C} X^{B} X_{A} X^{C}, \\
\delta A_{\mu}= & \frac{1}{2} \epsilon^{A B C D} X_{B} \bar{\Psi}_{A} \gamma_{\mu} \eta_{C D}+\bar{\Psi}^{A} \gamma_{\mu} \eta_{A B} X^{B}, \\
\delta \hat{A}_{\mu}= & \frac{1}{2} \epsilon^{A B C D} \bar{\Psi}_{A} \gamma_{\mu} \eta_{C D} X_{B}+X^{B} \bar{\Psi}_{A} \gamma_{\mu} \eta_{A B} .
\end{aligned}
$$

The supersymmetry transformations of $X^{C} X_{D}, X_{D} X^{C}$, and $\mathcal{M}$ are

$$
\begin{aligned}
\delta\left(X^{C} X_{D}\right) & =-\frac{i}{2} \epsilon^{C A B E} \bar{\eta}_{B E} \Psi_{A} X_{D}+i X^{C} \bar{\Psi}^{B} \eta_{D B}, \\
\delta\left(X_{D} X^{C}\right) & =-\frac{i}{2} \epsilon^{C A B E} \bar{\eta}_{B E} X_{D} \Psi_{A}+i \bar{\Psi}^{B} X^{C} \eta_{D B}, \\
\delta \mathcal{M} & =0
\end{aligned}
$$

For the given $V_{C D}$ or $U^{C D}$, one can always find the suitable $W_{B}^{A}$ and $\eta$ with

$$
\delta\left(W_{B}^{A} \mathcal{M}\right)=i\left(V_{C D} \mathcal{M}\right)
$$

or

$$
\delta\left(W_{B}^{A} \mathcal{M}\right)=i\left(U^{C D} \mathcal{M}\right) .
$$

So, 


$$
\begin{aligned}
& W_{B}^{A} \mathcal{M}=0 \Rightarrow V_{C D} \mathcal{M}=0, \\
& W_{B}^{A} \mathcal{M}=0 \Rightarrow U^{C D} \mathcal{M}=0 .
\end{aligned}
$$

It remains to be shown that $W_{D}^{C} \mathcal{M}=0$. The operator realization of $W_{D}^{C}$ is $-i \mathcal{K}_{D}^{C}$, with

$$
\begin{aligned}
\mathcal{K}_{D}^{C}= & \int d^{2} x \operatorname{tr}\left[\left(\Pi^{B} X_{B}-X^{B} \Pi_{B}\right) X^{C} X_{D}+\left(\Pi_{B} X^{B}-X_{B} \Pi^{B}\right) X_{D} X^{C}\right. \\
& \left.-\frac{k}{2 \pi} \epsilon^{i j} D_{i} X^{C} D_{j} X_{D}-\frac{i k}{2 \pi}\left(\bar{\Psi}^{B} \gamma_{0} \Psi_{B} X_{D} X^{C}+\bar{\Psi}_{B} \gamma_{0} \Psi^{B} X^{C} X_{D}\right)\right] .
\end{aligned}
$$

For example,

$$
\begin{aligned}
-i\left[\mathcal{K}_{D}^{C}, X^{B}\right] & =X^{C} X_{D} X^{B}-X^{B} X_{D} X^{C}, \\
-i\left[\mathcal{K}_{D}^{C}, A_{i}\right] & =-i D_{i}\left(X_{D} X^{C}\right) .
\end{aligned}
$$

$\mathcal{K}_{D}^{C}$ is gauge invariant and does not contain $F$, so $M_{R}(x) \mathcal{K}_{D}^{C} M_{R}^{-1}(x)=0,\left[M_{R}(x), \mathcal{K}_{D}^{C}\right]=0$, and

$$
W_{D}^{C} \mathcal{M}=-i\left[\mathcal{K}_{D}^{C}, \mathcal{M}\right]=0 .
$$

Up to now, Eq. (9.10) is verified.

In ABJM theory, from the SUSY invariance of the monopole operator, we can arrive at a conclusion similar to that of Eq. (9.10). In superspace formulation of the gauge theory, commutators of the covariant derivatives in superspace yield the field strengths, on which the constraints can be imposed. Supersymmetric gauge theories are entirely characterized by these constraints [36]. For ABJM theory in $\mathcal{N}=6$ superspace parametrized by the coordinates $\left(x^{\mu}, \theta^{I}\right)$, covariant derivatives should satisfy the constraints [26]

$$
\left\{D^{I}, \bar{D}^{J}\right\}=2 i \delta^{I J} \gamma^{\mu} D_{\mu}+i \mathcal{F}^{I J},
$$

where $D^{I}$ is the covariant derivative along $\theta^{I}, D_{\mu}$ is the covariant derivative in $3 \mathrm{D}$ spacetime, and $\mathcal{F}^{I J}=-\mathcal{F}^{J I}$ is the $I J$ component of the field strength in superspace whose lowest $\theta$ expansion is $\Sigma_{C}^{I J D}\left(X^{C} X_{D}\right)_{\hat{b}}^{\hat{a}}$ and $\Sigma_{C}^{I J D}\left(X_{D} X^{C}\right)_{b}^{a}$.

$$
\Sigma_{C}^{I J D}=\Gamma_{C A}^{[I} \tilde{\Gamma}^{J] A D}, \quad\left(\Sigma_{C}^{I J D}\right)^{*}=-\Sigma_{D}^{I J C} .
$$

$\Sigma_{C}^{I J C}=0$, so effectively,

$$
\begin{aligned}
& \Sigma_{C}^{I J D} X^{C} X_{D} \sim X^{C} X_{D}-\frac{1}{4} \delta_{D}^{C} X^{B} X_{B} \\
& \Sigma_{C}^{I J D} X_{D} X^{C} \sim X_{D} X^{C}-\frac{1}{4} \delta_{D}^{C} X_{B} X^{B} .
\end{aligned}
$$

The operator realization of $D^{I}$ and $\bar{D}^{J}$ is $\mathcal{Q}^{I}$ and $\overline{\mathcal{Q}}^{J}$. One may compute the anticommutator of supercharges directly:

$$
\left\{\mathcal{Q}^{I}, \overline{\mathcal{Q}}^{J}\right\}=-2 \delta^{I J} \gamma^{\mu} \mathcal{P}_{\mu}+\Sigma_{C}^{I J D} \mathcal{K}_{D}^{C}
$$

$\mathcal{P}_{\mu}$ is the gauge-invariant energy-momentum operator of ABJM theory which acts on fields as $-i D_{\mu}$. With $\mathcal{K}_{D}^{C}$ given by Eq. (9.21), the action of $\Sigma_{C}^{I J D} \mathcal{K}_{D}^{C}$ is the gauge variation $i \Sigma_{C}^{I J D} W_{D}^{C}$. When acting on gauge-invariant operators, Eq. (9.27) reduces to the standard supersymmetry algebra

$$
\left\{\mathcal{Q}^{I}, \overline{\mathcal{Q}}^{J}\right\}=-2 \delta^{I J} \gamma^{\mu} P_{\mu},
$$

with $P_{\mu}$ being the energy-momentum operator. $\left[\mathcal{Q}^{I}, \mathcal{M}\right]=0$, so $\left[\left\{\mathcal{Q}^{I}, \overline{\mathcal{Q}}^{J}\right\}, \mathcal{M}\right]=0$. From Eq. (9.27), when $I=J$, we get $D_{\mu} \mathcal{M}=0$; when $I \neq J$, we have $\Sigma_{C}^{I J D} W_{D}^{C} \mathcal{M}=0$, which is actually a weaker statement compared with $W_{D}^{C} \mathcal{M}=0$.

The anticommutator of $\mathcal{Q}^{I}$ and $\overline{\mathcal{S}}^{J}$ gives more superconformal charges. For example, from $\left\{\mathcal{Q}^{I}, \overline{\mathcal{S}}^{I}\right\}$, we may get $\mathcal{D}$, which is the gauge-invariant completion of the dilation $D$ and would reduce to $D$ when acting on gauge invariants. Since $\left[\mathcal{Q}^{I}, \mathcal{M}_{R}\right]=\left[\mathcal{S}^{J}, \mathcal{M}_{R}\right]=0, \quad\left[\mathcal{D}, \mathcal{M}_{R}\right]=0$. $\mathcal{M}_{R}$ has the conformal dimension 0. In Sec. VI, for $\mathcal{M}_{R}$ to have the conformal dimension 0 under the action of $D, a_{i}$ must take the special form in Eq. (6.5), which is not necessary when considering the gauge invariants.

Usually, the current conservation equation may impose some constraints on the classical Lagrangian. For example, $\partial^{\mu} j_{\mu B}^{A}=0$ requires that the Lagrangian be $S U(4)$ invariant. To study the constraints imposed by $\partial^{\mu} j_{\mu}^{A B}=0$, we consider a truncated ABJM model with no spinor fields for simplicity, where the monopole operators satisfy $D_{\mu} \mathcal{M}=0$ and $W_{D}^{C} \mathcal{M}=0$. Among all of the $S U(4)$ invariant scalar potentials, only the mass term $m^{2} \operatorname{tr}\left(X^{A} X_{A}\right)$ and the sextic potential in Eq. (7.14) could make $j_{\mu}^{A B}$ conserved. The requirement of symmetry enhancement almost fixes the interaction potential.

From the conserved currents in Eqs. (9.1) and (9.3), $15+$ $6+6=27$ conserved R-symmetry charges 


$$
\begin{gathered}
R_{B}^{A}=-\frac{i k}{2 \pi} \int d^{2} y\left\{\left[X_{a}^{A \hat{a}}\left(D_{0} X_{B}\right)_{\hat{a}}^{a}-\left(D_{0} X^{A}\right)_{a}^{\hat{a}} X_{B \hat{a}}^{a}+i \bar{\Psi}_{\hat{a}}^{A a} \gamma_{0} \Psi_{B a}^{\hat{a}}\right]\right. \\
\left.-\frac{1}{4} \delta_{B}^{A}\left[X_{a}^{C \hat{a}}\left(D_{0} X_{C}\right)_{\hat{a}}^{a}-\left(D_{0} X^{C}\right)_{a}^{\hat{a}} X_{C \hat{a}}^{a}+i \bar{\Psi}_{\hat{a}}^{C a} \gamma_{0} \Psi_{C a}^{\hat{a}}\right]\right\}, \\
R^{A B}=-\frac{i k}{4 \pi} \int d^{2} y \mathcal{M}_{\hat{a} \hat{b}}^{a b}\left[X_{a}^{A \hat{a}}\left(D_{0} X^{B}\right)_{b}^{\hat{b}}-\left(D_{0} X^{A}\right)_{a}^{\hat{a}} X_{b}^{B \hat{b}}+\frac{i}{2} \epsilon^{A B C D \bar{\Psi}_{C a}^{\hat{a}} \gamma_{0}} \Psi_{D b}^{\hat{b}}\right],
\end{gathered}
$$

and

$$
R_{A B}=R^{A B+}=\frac{i k}{4 \pi} \int d^{2} y \mathcal{M}_{a b}^{\hat{a} \hat{b}}\left[X_{A \hat{a}}^{a}\left(D_{0} X_{B}\right)_{\hat{b}}^{b}-\left(D_{0} X_{A}\right)_{\hat{a}}^{a} X_{B \hat{b}}^{b}-\frac{i}{2} \epsilon_{A B C D} \bar{\Psi}_{\hat{a}}^{C a} \gamma_{0} \Psi_{\hat{b}}^{D b}\right]
$$

are obtained, which, together with Eq. (3.17), compose the generators of the $S O(8)$ group. The commutation relations of the R-symmetry charges obey the so(8) algebra. In particular,

$\left[R^{A B}, R_{C D}\right]=\frac{1}{4}\left[\delta_{D}^{A} R_{C}^{B}-\delta_{C}^{A} R_{D}^{B}+\delta_{C}^{B} R_{D}^{A}-\delta_{D}^{B} R_{C}^{A}\right]$

requires $\mathcal{M}_{\hat{a} \hat{b}}^{a b} \mathcal{M}_{c b}^{\hat{c} \hat{b}}=\delta_{c}^{a} \delta_{\hat{a}}^{\hat{c}}$, which is proved in Eq. (5.27).

\section{X. $\mathcal{N}=8$ SUPERSYMMETRY IN ABJM THEORY}

The dynamical fields of the ABJM theory consist of $X_{A \hat{a}}^{a}$, $\Psi_{A a}^{\hat{a}}$ in the $\overline{4}$ representation and the adjoints $X_{a}^{A \hat{a}}, \Psi_{\hat{a}}^{A a}$ in the 4 representation of the $S U(4) \mathrm{R}$ symmetry. Although there are eight scalars $\left(X_{A}, X^{A}\right)$ and eight spinors $\left(\Psi_{A}, \Psi^{A}\right)$, they cannot be transformed into each other by the $S O(8)$ rotations due to the distinct gauge group representations. With the nondynamical Chern-Simons gauge fields $A_{\mu b}^{a}$ and $\hat{A}_{\mu \hat{b}}^{\hat{a}}$ added, the monopole operator $\mathcal{M}_{R}(x)$ can be obtained. In particular, using $\mathcal{M}_{\hat{a} \hat{b}}^{a b}(x)$ and $\mathcal{M}_{a b}^{\hat{a} \hat{b}}(x)$, one may construct the dressed fields [12,24]

$\tilde{X}_{\hat{a}}^{A a}(x)=\mathcal{M}_{\hat{a} \hat{b}}^{a b}(x) X_{b}^{A \hat{b}}(x), \quad \tilde{\Psi}_{A \hat{a}}^{a}(x)=\mathcal{M}_{\hat{a} \hat{b}}^{a b}(x) \Psi_{A b}^{\hat{b}}(x)$,

with the adjoints

$\tilde{X}_{A a}^{\hat{a}}(x)=\mathcal{M}_{a b}^{\hat{a} \hat{b}}(x) X_{A \hat{b}}^{b}(x), \quad \tilde{\Psi}_{a}^{A \hat{a}}(x)=\mathcal{M}_{a b}^{\hat{a} \hat{b}}(x) \Psi_{\hat{b}}^{A b}(x)$.

The eight scalars $\left(X_{A}, \tilde{X}^{A}\right)$ and the eight spinors $\left(\tilde{\Psi}_{A}, \Psi^{A}\right)$ are now in the same representation of the gauge group, and thus could be transformed into each other by the $S O(8)$ rotations.

Except for modifying the gauge group representation, $\mathcal{M}_{R}(x)$ 's are "silent" in several respects. They have the weight 0 , and thus would not change the dressed operator's conformal dimension; they commute with the supercharges, and thus would not influence the dressed operator's supersymmetry; they are covariantly constant, and thus would not affect the dressed operator's covariant derivative. This is different from the monopole operator in 3D Yang-Mills matter theories where the gauge fields are dynamical.

The $S O(8)$ symmetry group has $28=15+6 \times 2+1$ real parameters. The first 15 are $\omega_{A}^{B}$ 's satisfying $\left(\omega_{B}^{A}\right)^{*}+\omega_{A}^{B}=0, \quad \omega_{A}^{A}=0 . \quad \omega_{A}^{B} R_{B}^{A}$ generates the $S U(4)$ transformation, under which

$$
\begin{array}{ll}
\delta X_{A}=\omega_{A}^{B} X_{B}, & \delta \tilde{X}^{A}=-\omega_{B}^{A} \tilde{X}^{B}, \\
\delta \tilde{\Psi}_{A}=\omega_{A}^{B} \tilde{\Psi}_{B}, & \delta \Psi^{A}=-\omega_{B}^{A} \Psi^{B},
\end{array}
$$

and

$$
\begin{array}{ll}
\delta X^{A}=-\omega_{B}^{A} X^{B}, & \delta \tilde{X}_{A}=\omega_{A}^{B} \tilde{X}_{B}, \\
\delta \tilde{\Psi}^{A}=-\omega_{B}^{A} \tilde{\Psi}^{B}, & \delta \Psi_{A}=\omega_{A}^{B} \Psi_{B}
\end{array}
$$

for adjoints. The $6 \times 2$ real parameters are $\omega^{A B}$,s satisfying

$$
\omega^{A B}+\omega^{B A}=0, \quad\left(\omega^{A B}\right)^{*}+\omega_{B A}=0 .
$$

$R_{A B} \omega^{A B}$ and $R^{A B} \omega_{A B}$ generate the transformations

$\delta X_{A}=\omega_{A B} \tilde{X}^{B}, \quad \delta \tilde{X}^{A}=\omega^{A B} X_{B}$,

$\delta \tilde{\Psi}_{A}=\frac{1}{2} \epsilon_{A B C D} \omega^{B C} \Psi^{D}, \quad \delta \Psi^{A}=\frac{1}{2} \epsilon^{A B C D} \omega_{B C} \tilde{\Psi}_{D}$,

and

$$
\begin{aligned}
& \delta X^{A}=\omega^{A B} \tilde{X}_{B}, \quad \delta \tilde{X}_{A}=\omega_{A B} X^{B}, \\
& \delta \tilde{\Psi}^{A}=\frac{1}{2} \epsilon^{A B C D} \omega_{B C} \Psi_{D}, \quad \delta \Psi_{A}=\frac{1}{2} \epsilon_{A B C D} \omega^{B C} \tilde{\Psi}^{D}
\end{aligned}
$$


for adjoints. For Eqs. (10.6) and (10.7) to be consistent with Eqs. (10.1) and (10.2),

$$
\mathcal{M}_{\hat{a} \hat{b}}^{a b}(x) \mathcal{M}_{a c}^{\hat{a} \hat{c}}(x)=\delta_{c}^{b} \delta_{\hat{b}}^{\hat{c}}
$$

is again needed.

Aside from the monopole dressed matter fields, one may also introduce the monopole dressed gauge fields $\tilde{A}_{\mu a}^{c}$ and $\tilde{\hat{A}}_{\mu \hat{a}}^{\hat{c}}$ with

$$
\begin{aligned}
& \tilde{A}_{\mu c}^{a}=\mathcal{M}_{c d} A_{\mu b}^{d} \mathcal{M}^{b a}+i \mathcal{M}_{c b} \partial_{\mu} \mathcal{M}^{b a}, \\
& \tilde{\hat{A}}_{\mu \hat{a}}^{\hat{c}}=\mathcal{M}_{\hat{a} \hat{b}} \hat{A}_{\mu \hat{d}}^{\hat{b}} \mathcal{M}^{\hat{d} \hat{c}}+i \mathcal{M}_{\hat{a} \hat{b}} \partial_{\mu} \mathcal{M}^{\hat{b} \hat{c}}
\end{aligned}
$$

so that

$$
\begin{array}{r}
\left(\tilde{D}_{\mu} \tilde{X}^{B}\right)_{\hat{a}}^{a}=\partial_{\mu} \tilde{X}_{\hat{a}}^{B a}+i\left(\tilde{A}_{\mu c}^{a} \tilde{X}_{\hat{a}}^{B c}-\tilde{X}_{\hat{c}}^{B a} \tilde{\hat{A}}_{\mu \hat{a}}^{\hat{c}}\right)=\mathcal{M}_{\hat{a} \hat{b}}^{a b}\left(D_{\mu} X^{B}\right)_{b}^{\hat{b}}, \\
\left(\tilde{D}_{\mu} \tilde{X}_{B}\right)_{a}^{\hat{a}}=\partial_{\mu} \tilde{X}_{B a}^{\hat{a}}+i\left(\tilde{\hat{A}}_{\mu \hat{c}}^{\hat{a}} \tilde{X}_{B a}^{\hat{c}}-\tilde{X}_{B c}^{\hat{a}} \tilde{A}_{\mu a}^{c}\right)=\mathcal{M}_{a b}^{\hat{a} \hat{b}}\left(D_{\mu} X_{B}\right)_{\hat{b}}^{b},
\end{array}
$$

where we have used

$$
\begin{aligned}
\mathcal{M}_{\hat{a} \hat{b}}^{a b} & =\mathcal{M}^{a b} \mathcal{M}_{\hat{a} \hat{b}}, \quad \mathcal{M}_{a b}^{\hat{a} \hat{b}}=\mathcal{M}_{a b} \mathcal{M}^{\hat{a} \hat{b}}, \\
\mathcal{M}_{a c} \mathcal{M}^{c b} & =\delta_{a}^{b}, \quad \mathcal{M}_{\hat{a} \hat{c}} \mathcal{M}^{\hat{c} \hat{b}}=\delta_{\hat{a}}^{\hat{b}} .
\end{aligned}
$$

With the fields replaced by the monopole dressed fields, the ABJM action [Eq. (7.10)] is invariant:

$$
\begin{aligned}
& S\left(X_{A \hat{a}}^{a}, X_{a}^{A \hat{a}}, \Psi_{A a}^{\hat{a}}, \Psi_{\hat{a}}^{A a}, A_{b}^{a}, \hat{A}_{\hat{b}}^{\hat{a}}\right) \\
& =S\left(\tilde{X}_{A a}^{\hat{a}}, \tilde{X}_{\hat{a}}^{A a}, \tilde{\Psi}_{A \hat{a}}^{a}, \tilde{\Psi}_{a}^{A \hat{a}}, \tilde{A}_{a}^{b}, \tilde{\hat{A}}_{\hat{a}}^{\hat{b}}\right) .
\end{aligned}
$$

Moreover, $D_{\mu} \mathcal{M}^{b a}=D_{\mu} \mathcal{M}^{\hat{b} \hat{c}}=0$ is equivalent to the conditions

$$
\tilde{A}_{\mu c}^{a}=-A_{\mu c}^{a}, \quad \tilde{\hat{A}}_{\mu \hat{a}}^{\hat{c}}=-\hat{A}_{\mu \hat{a}}^{\hat{c}}
$$

so the gauge fields are invariant under the monopole dressing if and only if the monopole operators are covariantly constant.

With the R charges $R^{A B}$ and $R_{A B}$ added into the $\mathcal{N}=6$ superconformal algebra, two additional supercharges will be generated. For convenience, we will use

$$
\mathcal{R}^{I}=\Gamma_{A B}^{I} R^{A B}, \quad \mathcal{R}^{I+}=-\tilde{\Gamma}^{I A B} R_{A B}
$$

instead of $R^{A B}$ and $R_{A B}$. The commutator of $\mathcal{Q}^{I}$ and $\mathcal{R}^{J}$ gives two extra supercharges $\mathcal{Q}$ and the adjoint $\mathcal{Q}^{+}$:

$$
\left[\mathcal{R}^{J}, \mathcal{Q}^{I}\right]=\delta^{I J} \mathcal{Q}, \quad\left[\mathcal{R}^{J+}, \mathcal{Q}^{I}\right]=-\delta^{I J} \mathcal{Q}^{+},
$$

where

$$
\begin{aligned}
\mathcal{Q}= & -\frac{k}{2 \pi} \int d^{2} x \operatorname{tr}\left[\left(2 \gamma^{\mu} D_{\mu} \tilde{X}^{D}+X_{A} X^{A} \tilde{X}^{D}\right.\right. \\
& \left.\left.-\tilde{X}^{D} X^{A} X_{A}\right) \gamma_{0} \Psi_{D}+\frac{4}{3} \epsilon_{A B C D} X^{A} \tilde{X}^{B} X^{C} \gamma_{0} \Psi^{D}\right] .
\end{aligned}
$$

To get Eqs. (10.16) and (10.17), we have used the properties of Eq. (9.10) and $\left[\mathcal{Q}^{I}, \mathcal{M}\right]=0$. Also, because of Eq. (9.10), the right-hand side of Eq. (10.17) can take several different but equivalent forms.

For example, using $U^{C D} \mathcal{M}=0$, we have

$$
\begin{gathered}
\operatorname{tr}\left[\left(X^{B} \tilde{X}^{A} X^{C}-X^{C} \tilde{X}^{A} X^{B}\right) \gamma_{0} \Psi^{M}\right] \\
=\operatorname{tr}\left[\left(X^{C} \tilde{X}^{B} X^{A}-X^{A} \tilde{X}^{B} X^{C}\right) \gamma_{0} \Psi^{M}\right] \\
=\operatorname{tr}\left[\left(X^{A} \tilde{X}^{C} X^{B}-X^{B} \tilde{X}^{C} X^{A}\right) \gamma_{0} \Psi^{M}\right] \\
=\frac{1}{3} \epsilon^{B A C M} \epsilon_{P Q R M} \operatorname{tr}\left[\left(X^{P} \tilde{X}^{Q} X^{R}\right) \gamma_{0} \Psi^{M}\right],
\end{gathered}
$$

so effectively,

$X^{B} \tilde{X}^{A} X^{C}-X^{C} \tilde{X}^{A} X^{B} \sim \frac{1}{3} \epsilon^{B A C M} \epsilon_{P Q R M}\left(X^{P} \tilde{X}^{Q} X^{R}\right) \sim \overline{4}$

is in the $\overline{4}$ representation of $S U(4)$. In Ref. [26], the constraint $X^{B} \tilde{X}^{A} X^{C}-X^{C} \tilde{X}^{A} X^{B} \sim \overline{4}$ was derived in Eq. (6.11) as a consequence of $D_{\mu} \mathcal{M}=0$. We have seen that such a condition can indeed be satisfied.

$\mathcal{Q}, \mathcal{Q}^{+}$, and $\mathcal{Q}^{I}$ altogether comprise eight supercharges for the $\mathcal{N}=8$ supersymmetry. Under the action of $\overline{\mathcal{Q}} \varepsilon$,

$\delta X_{D}=2 i \overline{\tilde{\Psi}}_{D} \varepsilon, \quad \delta X^{D}=0$,

$\delta \Psi^{D}=-2 \gamma^{\mu} D_{\mu} \tilde{X}^{D} \varepsilon+\left(X_{A} X^{A} \tilde{X}^{D}-\tilde{X}^{D} X^{A} X_{A}\right) \varepsilon$,

$\delta \Psi_{D}=-\frac{4}{3} \epsilon_{A B C D} X^{A} \tilde{X}^{B} X^{C} \varepsilon$

$$
\delta A_{i}=2 \tilde{X}^{D} \bar{\Psi}_{D} \gamma_{i} \varepsilon, \quad \delta \hat{A}_{i}=-2 \bar{\Psi}_{D} \gamma_{i} \varepsilon \tilde{X}^{D},
$$

and similarly for $\overline{\mathcal{Q}}^{+} \mathcal{\varepsilon}^{+}$. In Eq. (10.17), $\mathcal{Q}$ and $\mathcal{Q}^{+}$do not contain $F$, and thus should commute with $M_{R}(x)$. $\left[\mathcal{Q}, M_{R}(x)\right]=\left[\mathcal{Q}^{+}, M_{R}(x)\right]=0, \quad\left[\mathcal{Q}, \mathcal{M}_{R}(x)\right]=$ $\left[\mathcal{Q}^{+}, \mathcal{M}_{R}(x)\right]=0$, and $\mathcal{M}_{R}(x)$ is invariant under the $\mathcal{N}=8$ supersymmetry.

From Eq. (9.27), Eq. (10.16), and the so(8) algebra, we also have

$$
\begin{aligned}
{\left[\mathcal{R}^{I}, \mathcal{Q}\right] } & =0, & & {\left[\mathcal{R}^{I+}, \mathcal{Q}\right]=-2 \mathcal{Q}^{I}, } \\
{\left[\mathcal{R}^{I+}, \mathcal{Q}^{+}\right] } & =0, & & {\left[\mathcal{R}^{I}, \mathcal{Q}^{+}\right]=2 \mathcal{Q}^{I}, }
\end{aligned}
$$




$$
\begin{aligned}
\left\{\mathcal{Q}, \overline{\mathcal{Q}}^{I}\right\} & =\mathcal{K}^{I}, \quad\left\{\mathcal{Q}^{+}, \overline{\mathcal{Q}}^{I}\right\}=\mathcal{K}^{I+}, \\
\left\{\mathcal{Q}, \overline{\mathcal{Q}}^{+}\right\} & =4 \gamma^{\mu} \mathcal{P}_{\mu}+\mathcal{K},
\end{aligned}
$$

where $\mathcal{K}^{I}$ is a gauge variation with $6+6$ parameters $2 \Gamma_{M C}^{I} \tilde{X}^{M} X^{C}$ and $2 \Gamma_{M C}^{I} X^{C} \tilde{X}^{M}$,

$$
\left[\mathcal{K}^{I}, X_{A}\right]=2 i \Gamma_{M C}^{I}\left(\tilde{X}^{M} X^{C} X_{A}-X_{A} X^{C} \tilde{X}^{M}\right)
$$

$\mathcal{K}^{I+}$ is a gauge variation with $6+6$ parameters $-2 \tilde{\Gamma}^{I M C} X_{M} \tilde{X}_{C}$ and $-2 \tilde{\Gamma}^{I M C} \tilde{X}_{C} X_{M}$,

$\left[\mathcal{K}^{I+}, X_{A}\right]=-2 i \tilde{\Gamma}^{I M C}\left(X_{M} \tilde{X}_{C} X_{A}-X_{A} \tilde{X}_{C} X_{M}\right) ;$

and $\mathcal{K}$ is a gauge variation with $1+1$ parameters $2\left(X_{C} X^{C}-\tilde{X}^{C} \tilde{X}_{C}\right)$ and $2\left(X^{C} X_{C}-\tilde{X}_{C} \tilde{X}^{C}\right)$,

$$
\left[\mathcal{K}, X_{A}\right]=2 i\left[\left(X_{C} X^{C}-\tilde{X}^{C} \tilde{X}_{C}\right) X_{A}-X_{A}\left(X^{C} X_{C}-\tilde{X}_{C} \tilde{X}^{C}\right)\right] .
$$

When acting on the gauge-invariant operators, Eq. (10.26) becomes

$$
\left\{\mathcal{Q}, \overline{\mathcal{Q}}^{I}\right\}=0, \quad\left\{\mathcal{Q}^{+}, \overline{\mathcal{Q}}^{I}\right\}=0, \quad\left\{\mathcal{Q}, \overline{\mathcal{Q}}^{+}\right\}=4 \gamma^{\mu} P_{\mu}
$$

completing the $\mathcal{N}=8$ superalgebra.

In Ref. [32], a systematic classification of the unitary superconformal multiplets in $d \geq 3$ spacetime dimensions is given. In the following, specified to the ABJM model, we will give the explicit operator content for the $1 / 3$ BPS stress tensor multiplet, the $1 / 2$ BPS extra SUSY current

$$
[0]_{1}^{(0,1,1)} \stackrel{Q}{\longrightarrow}[1]_{\frac{3}{2}}^{(0,0,2) \oplus(0,2,0) \oplus(1,0,0)} \stackrel{Q}{\longrightarrow}[0]_{2}^{(0,1,1)}
$$

In $\mathrm{ABJM}$ theory, $[0]_{1}^{(0,1,1)}$ are 15 scalars $\operatorname{tr}\left(X^{A} X_{B}-\right.$ $\left.\frac{1}{4} \delta_{B}^{A} X^{C} X_{C}\right)$ annihilated by two supercharges. The action of the other four supercharges gives the whole multiplet. The $1+15$ dimension- 2 vectors $[2]_{2}^{(0,0,0) \oplus(0,1,1)}$ are the $U(1)_{J}$ current and the $S U(4)$ R-symmetry current $j_{\mu B}^{A}$ in Eq. (9.1). [3] $]_{\frac{5}{2}}^{(1,0,0)}$ is the SUSY current whose integration gives the supercharges $\mathcal{Q}^{I}$. $[4]_{3}^{(0,0,0)}$ is the stress tensor.

Sometimes, the theory also contains a $32+321 / 2$ BPS extra SUSY-current multiplet with chiral primary operators in the $[0]_{1}^{(0,0,2)}$ representation. The whole multiplet is given by

$$
[0]_{1}^{(0,0,2)} \stackrel{Q}{\longrightarrow}[1]_{\frac{3}{2}}^{(0,1,1)} \stackrel{Q}{\longrightarrow}[0]_{2}^{(0,2,0)} \oplus[2]_{2}^{(1,0,0)} \stackrel{Q}{\longrightarrow}[3]_{\frac{5}{2}}^{(0,0,0)},
$$

multiplet, the 1/6 BPS higher-spin current multiplet, the free hypermultiplet of the $3 \mathrm{D} \mathcal{N}=6$ SCFT, and the $1 / 2$ BPS stress tensor multiplet of the $3 \mathrm{D} \mathcal{N}=8$ SCFT. The operators are characterized by $[2 j]_{\Delta}^{(R)}$, where $j$ is the halfinteger $s u(2)$ spin, $\Delta$ is the conformal dimension, and $R$ stands for the Dynkin labels of the R-symmetry representation [32].

We will take

$$
\overline{\mathcal{Q}}^{A B}=-\frac{1}{4} \bar{Q}^{I} \tilde{\Gamma}^{I A B}
$$

instead of $\overline{\mathcal{Q}}^{I}$ for $\mathcal{N}=6$ supercharges. Then

$$
\begin{aligned}
& {\left[\overline{\mathcal{Q}}^{A B} \eta_{A B}, X_{C}\right]=i \bar{\Psi}^{B} \eta_{C B},} \\
& {\left[\overline{\mathcal{Q}}^{A B} \eta_{A B}, X^{C}\right]=-\frac{i}{2} \epsilon^{C D M N} \bar{\Psi}_{D} \eta_{M N},} \\
& {\left[\overline{\mathcal{Q}}^{A B} \eta_{A B}, \tilde{X}_{C}\right]=i \overline{\tilde{\Psi}}^{B} \eta_{C B},} \\
& {\left[\overline{\mathcal{Q}}^{A B} \eta_{A B}, \tilde{X}^{C}\right]=-\frac{i}{2} \epsilon^{C D M N} \overline{\tilde{\Psi}}_{D} \eta_{M N},} \\
& {\left[\overline{\mathcal{Q}}, X^{C}\right]=0, \quad\left[\overline{\mathcal{Q}}, \tilde{X}^{C}\right]=0,} \\
& {\left[\overline{\mathcal{Q}}^{+}, X_{C}\right]=0, \quad\left[\overline{\mathcal{Q}}^{+}, \tilde{X}_{C}\right]=0,}
\end{aligned}
$$

where $\left[\overline{\mathcal{Q}}^{A B}, \mathcal{M}\right]=[\overline{\mathcal{Q}}, \mathcal{M}]=\left[\overline{\mathcal{Q}}^{+}, \mathcal{M}\right]=0$ is used.

According to Ref. [32], in 3D $\mathcal{N}=6$ SCFT, there is a protected 1/3 BPS stress tensor multiplet with chiral primary operators in the $[0]_{1}^{(0,1,1)}$ representation. Successive action of the supercharges gives the complete $64+64$ multiplet: with the complex conjugation

$[0]_{1}^{(0,2,0)} \stackrel{Q}{\longrightarrow}[1]_{\frac{3}{2}}^{(0,1,1)} \stackrel{Q}{\longrightarrow}[0]_{2}^{(0,0,2)} \oplus[2]_{2}^{(1,0,0)} \stackrel{Q}{\longrightarrow}[3]_{\frac{5}{2}}^{(0,0,0)}$.

In ABJM theory with $k=1,2,[0]_{1}^{(0,0,2)}$ and $[0]_{1}^{(0,2,0)}$ are ten scalars $\operatorname{tr}\left(X^{A} \tilde{X}^{B}\right)$ and the complex conjugate $\operatorname{tr}\left(X_{A} \tilde{X}_{B}\right)$. The highest-weight operator can be selected as $\operatorname{tr}\left(X^{1} \tilde{X}^{1}\right)$, which is annihilated by three supercharges $\overline{\mathcal{Q}}^{12}, \overline{\mathcal{Q}}^{13}$, and $\overline{\mathcal{Q}}^{14}$ based on Eqs. (10.32) and (10.33). The other three supercharges generate the whole multiplet. The six dimension-2 vectors $[2]_{2}^{(1,0,0)}$ are the extra R-symmetry current $j_{\mu}^{A B}$ in Eq. (9.3). The top component, $[3]_{\frac{5}{2}}^{(0,0,0)}$, is the extra SUSY-current whose integration gives the supercharge $\mathcal{Q}$ with the adjoint $\mathcal{Q}^{+}$. 
When the extra SUSY-current multiplets exist, the $\mathcal{N}=$ 6 theory actually has eight supercharges: $\overline{\mathcal{Q}}^{A B}, \overline{\mathcal{Q}}$, and $\overline{\mathcal{Q}}^{+}$. According to Ref. [32], the 3D $\mathcal{N}=8$ SCFT has a
$128+1281 / 2$ BPS stress tensor multiplet with $15+10+$ 10 chiral primary operators in the $[0]_{1}^{(0,0,0,2)}$ representation. The whole multiplet is given by

$$
[0]_{1}^{(0,0,0,2)} \stackrel{Q}{\longrightarrow}[1]_{\frac{3}{2}}^{(0,0,1,1)} \stackrel{Q}{\longrightarrow}[0]_{2}^{(0,0,2,0)} \oplus[2]_{2}^{(0,1,0,0)} \stackrel{Q}{\longrightarrow}[3]_{\frac{5}{2}}^{(1,0,0,0)} \stackrel{\mathcal{Q}}{\longrightarrow}[4]_{3}^{(0,0,0,0)}
$$

Here, $[0]_{1}^{(0,0,0,2)}$ is composed of 15 scalars $\operatorname{tr}\left(X^{A} X_{B}-\frac{1}{4} \delta_{B}^{A} X^{C} X_{C}\right), 10$ scalars $\operatorname{tr}\left(X^{A} \tilde{X}^{B}\right)$, and 10 scalars $\operatorname{tr}\left(X_{A} \tilde{X}_{B}\right)$, among which the highest-weight operator can be selected as $\operatorname{tr}\left(X^{1} \tilde{X}^{1}\right)$. The action of the $S O(8) \mathrm{R}$ charges gives the complete $[0]_{1}^{(0,0,0,2)}$ representation. From Eqs. (10.32)-(10.34), $\operatorname{tr}\left(X^{1} \tilde{X}^{1}\right)$ is a $1 / 2$ BPS operator annihilated by four supercharges: $\overline{\mathcal{Q}}^{12}, \overline{\mathcal{Q}}^{13}, \overline{\mathcal{Q}}^{14}$, and $\overline{\mathcal{Q}}$. The action of the other four supercharges gives the whole multiplet, among which $[2]_{2}^{(0,1,0,0)},[3]_{\frac{5}{2}}^{(1,0,0,0)}$, and $[4]_{3}^{(0,0,0,0)}$ are the $S O(8)$ R-symmetry current, the $\mathcal{N}=8$ SUSY current, and the stress tensor, which is just $[4]_{3}^{(0,0,0)}$ in $\mathcal{N}=6$ language.
The extra SUSY-current multiplets are built from $\mathcal{M}_{\hat{a} \hat{b}}^{a b}(x)$ and $\mathcal{M}_{a b}^{\hat{a} \hat{b}}(x)$, which is the only possibility when $k=2$. However, when $k=1$, it seems that $\mathcal{M}_{\hat{a} \hat{b}}^{\prime a b}(x)$ and $\mathcal{M}_{a b}^{\prime a \hat{b}}(x)$ in Eq. (5.28) will give another set of extra SUSYcurrent multiplets. The existence of two copies of $\mathcal{N}=8$ supercurrents in the $k=1 \mathrm{ABJM}$ model is discussed in Ref. [23].

Besides this, a 1/6 BPS higher-spin current multiplet with chiral primary operators in the $[0]_{1}^{(1,0,0)}$ representation is also allowed in $3 \mathrm{D} \mathcal{N}=6$ SCFT [32]. The whole multiplet is given by

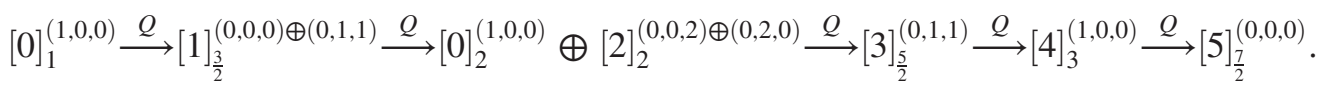

The scalars $\mathcal{M}_{\hat{a} \hat{b}}^{a b} X_{a}^{A \hat{a}} X_{b}^{B \hat{b}}$ are in the $[0]_{1}^{(0,0,2)}$ representation. When $k=1$, using $\mathcal{M}_{\hat{a} \hat{b}}^{\prime \prime a}$ or $\mathcal{M}_{\hat{a} \hat{b}}^{\prime \prime \prime a b}$ in Eqs. (5.29) and (5.30), $\mathcal{M}_{\hat{a} \hat{b}}^{\prime \prime a b} X_{a}^{A \hat{a}} X_{b}^{B \hat{b}}$ or $\mathcal{M}_{\hat{a} \hat{b}}^{\prime \prime \prime a b} X_{a}^{A \hat{a}} X_{b}^{B \hat{b}}$ in the representation $[0]_{1}^{(1,0,0)}$ can be obtained, which is annihilated by one supercharge. The other five supercharges generate the complete multiplet. From $\mathcal{M}_{\hat{a} \hat{b}}^{\prime \prime a b}\left(\mathcal{M}_{\hat{a} \hat{b}}^{\prime \prime \prime a b}\right)$, one can only construct the separate bosonic currents

$j_{\mu X}^{A B}=-\frac{i k}{4 \pi} \mathcal{M}_{\hat{a} \hat{b}}^{\prime \prime a b}\left[X_{a}^{A \hat{a}}\left(D_{\mu} X^{B}\right)_{b}^{\hat{b}}-\left(D_{\mu} X^{A}\right)_{a}^{\hat{a}} X_{b}^{B \hat{b}}\right]$

and fermionic currents

$$
j_{\mu A B \Psi}=\frac{k}{4 \pi} \mathcal{M}_{\hat{a} \hat{b}}^{\prime \prime a b}\left[\bar{\Psi}_{A a}^{\hat{a}} \gamma_{\mu} \Psi_{B b}^{\hat{b}}\right]
$$

corresponding to $[2]_{2}^{(0,0,2)}$ and $[2]_{2}^{(0,2,0)}$ in Eq. (10.39). Similarly to Appendix B, we may find that $j_{\mu X}^{A B}$ and $j_{\mu A B \Psi}$ are not conserved due to the fermionic interaction [Eq. (7.13)]. This is expected, since otherwise, we will get the conserved higher-spin current, which can only exist in free theory [37]. So, the multiplet in Eq. (10.39) is unprotected and would not bring the additional symmetry.

Finally, 3D $\mathcal{N}=6$ SCFT can also have the free hypermultiplets exchanged by complex conjugation [32]:

$$
[0]_{\frac{1}{2}}^{(0,1,0)} \stackrel{Q}{\longrightarrow}[1]_{1}^{(0,0,1)}, \quad[0]_{\frac{1}{2}}^{(0,0,1)} \stackrel{Q}{\longrightarrow}[1]_{1}^{(0,1,0)} .
$$

In the $k=1$ ABJM model, $\left\{[0]_{\frac{1}{2}}^{(0,1,0)},[0]_{\frac{1}{2}}^{(0,0,1)}\right\}$ and $\left\{[1]_{1}^{(0,0,1)}, \quad[1]_{1}^{(0,1,0)}\right\} \quad$ correspond to the scalars $\left\{x^{A}=\mathcal{M}_{\hat{a}}^{a} X_{a}^{A \hat{a}}, \quad x_{A}=\mathcal{M}_{a}^{\hat{a}} X_{A \hat{a}}^{a}\right\}, \quad$ and spinors $\left\{\psi_{A}=\mathcal{M}_{\hat{a}}^{a} \Psi_{A a}^{\hat{a}}, \quad \psi^{A}=\mathcal{M}_{a}^{\hat{a}} \Psi_{\hat{a}}^{A a}\right\}, \quad$ respectively. From Eqs. (9.11), (9.12), (B3), and (B4), using Eq. (9.10), we have

$$
\delta x_{A}=i \bar{\psi}^{B} \eta_{A B}, \quad \delta \psi_{A}=\gamma^{\mu} \eta_{A B} \partial_{\mu} x^{B}
$$

and

$$
\partial^{2} x_{A}=0, \quad \gamma^{\mu} \partial_{\mu} \psi_{A}=0 .
$$

So $(x, \psi)$ are indeed free. In $U(N)$ SYM theories, the decoupled free sector is the trace of the fundamental fields, but here the monopole operators must be used.

\section{CONCLUSION AND DISCUSSION}

The main result of the paper is composed of two parts. First, based on the original definition in Ref. [1], we constructed the monopole operators and computed the contraction relations, classical conformal dimensions, 
supersymmetry transformations, and covariant derivatives. Second, with the concrete form of the monopole operators given, we studied their role in the global symmetry enhancement of the ABJM theory and proved several assumptions that were made with them to achieve the symmetry enhancement.

In Chern-Simons matter theories with nondynamical gauge fields, monopole operators commute with the supercharges, the covariant derivative operators, and some particular field-dependent gauge variation operators. The ordinary gauge transformations also commute with these gauge-invariant operators, while the monopole operators are just the singular gauge transformations whose singularity does not have the manifestation here. As a result, except for changing the matter field's gauge representation, monopole operators do not have the side effect, when combined with the matter, which makes them suitable ingredients in the symmetry enhancement.

$5 \mathrm{D}$ gauge theories also contain a conserved topological current $J^{\mu}=\frac{1}{32 \pi^{2}} \epsilon^{\mu \nu \lambda \alpha \beta} \operatorname{tr}\left(F_{\nu \lambda} F_{\alpha \beta}\right)$. The conserved charge

$$
Q_{I}=\frac{1}{32 \pi^{2}} \int d^{4} y \epsilon^{i j k l} \operatorname{tr}\left(F_{i j} F_{k l}\right)
$$

is the instanton number, and the corresponding global symmetry is the $U(1)_{I}$ symmetry. As the higher-dimensional analogues of the 3D monopole operators, instanton operators are local disorder operators creating an instanton number on a $S^{4}$ surrounding their insertion point and could be defined by specifying the field configurations carrying the nonvanishing instanton number on $S^{4}$ [38-40]. Global symmetry enhancement may occur at the UV fixed point of the 5D gauge theories [41]. The original global symmetry algebra $\mathcal{R} \oplus Q_{I}$ of the theory can be enhanced to $\hat{\mathcal{R}}=$ $\mathcal{R} \oplus Q_{I} \oplus \mathcal{R}_{\text {off-diag }}$ if the currents for $\mathcal{R}_{\text {off-diag }}$ can be built from the instanton operators and are conserved [42-45]. In parallel with the discussion for monopole operators, one may write down the instanton operators, construct the $\mathcal{R}_{\text {off-diag }}$ currents, and investigate their conservation.

\section{ACKNOWLEDGMENTS}

The work is supported in part by NSFC under Grant No. 11605049.

\section{APPENDIX A: SOLITONS AND SOLITON OPERATORS}

In a $D$-dimensional gauge theory with the gauge group $G$, the finite-action gauge field configuration should satisfy

$$
A_{i} \rightarrow i u^{-1} \partial_{i} u, \quad u \in G
$$

at the spatial boundary $\partial R^{D-1} \cong S_{\infty}^{D-2}, i=1, \ldots, D-1$. So, the finite-action configurations provide maps from $S_{\infty}^{D-2}$ to $G$, which is labeled by the homotopy group
$\Pi_{D-2}(G)$. Two maps are in the same homotopy class if they can be continuously deformed into each other. The surface integral

$$
\int_{S_{\infty}^{D-2}} \operatorname{tr}\left[\left(u^{-1} d u\right)^{D-2}\right]
$$

counts how many times the group wraps itself around $S_{\infty}^{D-2}$ and is simply 0 when $\Pi_{D-2}(G)=0$.

When $D=3, G=U(N), 2 \mathrm{D}$ gauge fields are classified by the homotopy group $\Pi_{1}(U(N)) \cong \mathbb{Z}$. The integer $q \in \mathbb{Z}$ is just the vortex charge in Eq. (1.1):

$$
\begin{aligned}
Q & =\frac{1}{4 \pi} \int d^{2} x \epsilon^{i j} \operatorname{tr} F_{i j} \\
& =\frac{1}{2 \pi} \int_{S_{\infty}^{1}} d S_{i} \epsilon^{i j} \operatorname{tr} A_{j} \\
& =\frac{i}{2 \pi} \int_{S_{\infty}^{1}} d S_{i} \epsilon^{i j} \operatorname{tr}\left(u^{-1} \partial_{j} u\right) .
\end{aligned}
$$

If $\left|A_{i}\right\rangle$ is in a homotopy class labeled by $q$, then

$$
Q\left|A_{i}\right\rangle=q\left|A_{i}\right\rangle
$$

Vortex operators are "large" gauge transformations that could cause the states in different homotopy classes to transform into each other. Consider a gauge transformation $U$ with $U A_{i} U^{-1}=g^{-1} A_{i} g+i g^{-1} \partial_{i} g$; if

$$
\frac{i}{2 \pi} \int_{S_{\infty}^{1}} d S_{i} \epsilon^{i j} \operatorname{tr}\left(g^{-1} \partial_{j} g\right)=k,
$$

then

$U Q U^{-1}=Q+\frac{i}{2 \pi} \int_{S_{\infty}^{1}} d S_{i} \epsilon^{i j} \operatorname{tr}\left(g^{-1} \partial_{j} g\right)=Q+k$.

So, $[Q, U]=-k U . U$ is a vortex operator (monopole operator) carrying $-k$ vortex charges.

$$
Q U\left|A_{i}\right\rangle=(q-k) U\left|A_{i}\right\rangle
$$

and $U\left|A_{i}\right\rangle$ is in a homotopy class labeled by $q-k$. The ordinary gauge transformation should have $k=0$. When $k \neq 0, g$ must be singular in at least one point, which is the location of the vortex operator. Such a $g$ is gauge equivalent to $\Omega_{\vec{m}}(\omega)$ in Sec. II.

When $D=5, G=U(N)$, or $S U(N)$ and $N \geq 2$, 4D gauge fields are classified by the homotopy group $\Pi_{3}(G) \cong \mathbb{Z}$. The integer $q \in \mathbb{Z}$ is the instanton number [Eq. (11.1)] 


$$
\begin{aligned}
Q_{I} & =\frac{1}{32 \pi^{2}} \int d^{4} y \epsilon^{i j k l} \operatorname{tr}\left(F_{i j} F_{k l}\right) \\
& =\frac{1}{8 \pi^{2}} \epsilon^{i j k l} \int_{S_{\infty}^{3}} d^{3} S_{i} \operatorname{tr}\left[A_{j}\left(\partial_{k} A_{l}-\frac{2 i}{3} A_{k} A_{l}\right)\right] \\
= & \frac{1}{24 \pi^{2}} \epsilon^{i j k l} \int_{S_{\infty}^{3}} d^{3} S_{i}\left(u^{-1} \partial_{j} u\right)\left(u^{-1} \partial_{k} u\right)\left(u^{-1} \partial_{l} u\right) .
\end{aligned}
$$

For a gauge transformation $U$ with $U A_{i} U^{-1}=g^{-1} A_{i} g+i g^{-1} \partial_{i} g$, if

$\frac{1}{24 \pi^{2}} \epsilon^{i j k l} \int_{S_{\infty}^{3}} d^{3} S_{i}\left(g^{-1} \partial_{j} g\right)\left(g^{-1} \partial_{k} g\right)\left(g^{-1} \partial_{l} g\right)=k$,

then

$$
\begin{aligned}
U Q_{I} U^{-1} & =Q_{I}+\frac{1}{24 \pi^{2}} \epsilon^{i j k l} \int_{S_{\infty}^{3}} d^{3} S_{i}\left(g^{-1} \partial_{j} g\right)\left(g^{-1} \partial_{k} g\right)\left(g^{-1} \partial_{l} g\right) \\
& =Q_{I}+k
\end{aligned}
$$

with $\left[Q_{I}, U\right]=-k U . U$ is an instanton operator with the instanton number $-k$. For $\left|A_{i}\right\rangle$ in a homotopy class labeled by $q, U\left|A_{i}\right\rangle$ is in a homotopy class labeled by $q-k$. The ordinary gauge transformations have $k=0$. When $k \neq 0$, the action of $U$ must be singular in at least one point - that is, the location of the instanton operator.

\section{APPENDIX B: CURRENT CONSERVATION EQUATION OF $j_{\mu}^{A B}$}

The enhancement of the global symmetry from $S U(4) \times$ $U(1)_{J}$ to $S O(8)$ requires the conservation of the 12 currents

$$
\begin{aligned}
j_{\mu}^{A B}= & -\frac{i k}{4 \pi} \mathcal{M}_{\hat{a} \hat{b}}^{a b}\left[X_{a}^{A \hat{a}}\left(D_{\mu} X^{B}\right)_{b}^{\hat{b}}-\left(D_{\mu} X^{A}\right)_{a}^{\hat{a}} X_{b}^{B \hat{b}}\right. \\
& \left.+\frac{i}{2} \epsilon^{A B C D} \bar{\Psi} \bar{C}_{C a}^{\hat{a}} \gamma_{\mu} \Psi_{D b}^{\hat{b}}\right]
\end{aligned}
$$

and the adjoints $j_{\mu A B}$.

Direct calculation gives

$$
\begin{aligned}
\partial^{\mu} j_{\mu}^{A B}= & -\frac{i k}{4 \pi}\left(D^{\mu} \mathcal{M}\right)_{\hat{a} \hat{b}}^{a b}\left[X_{a}^{A \hat{a}}\left(D_{\mu} X^{B}\right)_{b}^{\hat{b}}-\left(D_{\mu} X^{A}\right)_{a}^{\hat{a}} X_{b}^{B \hat{b}}+\frac{i}{2} \epsilon^{A B C D} \bar{\Psi}_{C a}^{\hat{a}} \gamma_{\mu} \Psi_{D b}^{\hat{b}}\right] \\
& -\frac{i k}{4 \pi} \mathcal{M}_{\hat{a} \hat{b}}^{a b}\left[X_{a}^{A \hat{a}}\left(D^{2} X^{B}\right)_{b}^{\hat{b}}-\left(D^{2} X^{A}\right)_{a}^{\hat{a}} X_{b}^{B \hat{b}}+i \epsilon^{A B C D} \bar{\Psi}_{C a}^{\hat{a}} \gamma_{\mu}\left(D^{\mu} \Psi_{D}\right)_{b}^{\hat{b}}\right] .
\end{aligned}
$$

From the action in Eqs. (7.10)-(7.14), we have

$$
\frac{k}{2 \pi} D^{2} X^{B}=R_{1}^{B}+R_{2}^{B}+R_{3}^{B}
$$

with

$$
\begin{aligned}
R_{1}^{B}= & 2 i \epsilon^{B C D E} \bar{\Psi}_{C} X_{D} \Psi_{E}, \\
R_{2}^{B}= & i\left(\bar{\Psi}_{C} \Psi^{C} X^{B}-X^{B} \bar{\Psi}^{C} \Psi_{C}+2 X^{C} \bar{\Psi}^{B} \Psi_{C}-2 \bar{\Psi}_{C} \Psi^{B} X^{C}\right), \\
R_{3}^{B}= & 2\left(X^{B} X_{C} X^{D} X_{D} X^{C}+X^{C} X_{D} X^{D} X_{C} X^{B}-2 X^{C} X_{D} X^{B} X_{C} X^{D}\right) \\
& -\left(X^{B} X_{C} X^{C} X_{D} X^{D}+X^{C} X_{C} X^{D} X_{D} X^{B}-2 X^{C} X_{C} X^{B} X_{D} X^{D}\right),
\end{aligned}
$$

and

$$
\frac{k}{2 \pi} \epsilon^{A B C D} \gamma^{\mu} D_{\mu} \Psi_{D}=R_{1}^{A B C}+R_{2}^{A B C}
$$

with

$$
\begin{aligned}
& R_{1}^{A B C}=\epsilon^{A B C D}\left(X^{E} X_{E} \Psi_{D}-\Psi_{D} X_{E} X^{E}-2 X^{E} X_{D} \Psi_{E}+2 \Psi_{E} X_{D} X^{E}\right) \\
& R_{2}^{A B C}=2\left(X^{A} \Psi^{C} X^{B}-X^{A} \Psi^{B} X^{C}+X^{B} \Psi^{A} X^{C}-X^{B} \Psi^{C} X^{A}+X^{C} \Psi^{B} X^{A}-X^{C} \Psi^{A} X^{B}\right)
\end{aligned}
$$

With Eqs. (B3) and (B4) plugged in, the second line of Eq. (B2) becomes

$$
-\frac{i}{2}\left[\mathcal{M}\left(X^{[A} R_{1}^{B]}+X^{[A} R_{2}^{B]}+X^{[A} R_{3}^{B]}\right)+i \mathcal{M} \bar{\Psi}_{C}\left(R_{1}^{A B C}+R_{2}^{A B C}\right)\right] .
$$


We may introduce the gauge variation $W_{D}^{C}$, the action of which on $X_{a}^{B a ̂}$ is

$$
\left(W_{D}^{C} X^{B}\right)_{a}^{\hat{a}} \equiv\left(X^{C} X_{D}\right)_{\hat{b}}^{\hat{a}} X_{a}^{B \hat{b}}-X_{b}^{B \hat{a}}\left(X_{D} X^{C}\right)_{a}^{b} .
$$

$R_{3}^{B}$ could be written as

$$
R_{3}^{B}=2\left(W_{C}^{D}\left(W_{D}^{C} X^{B}\right)\right)-\left(W_{C}^{C}\left(W_{D}^{D} X^{B}\right)\right) .
$$

We have

$$
\begin{aligned}
\mathcal{M} X^{A} R_{3}^{B}= & 2 \mathcal{M} X^{A}\left(W_{C}^{D}\left(W_{D}^{C} X^{B}\right)\right)-\mathcal{M} X^{A}\left(W_{C}^{C}\left(W_{D}^{D} X^{B}\right)\right) \\
= & -2\left(W_{C}^{D} \mathcal{M}\right) X^{A}\left(W_{D}^{C} X^{B}\right)-2 \mathcal{M}\left(W_{C}^{D} X^{A}\right)\left(W_{D}^{C} X^{B}\right) \\
& +\left(W_{C}^{C} \mathcal{M}\right) X^{A}\left(W_{D}^{D} X^{B}\right)+\mathcal{M}\left(W_{C}^{C} X^{A}\right)\left(W_{D}^{D} X^{B}\right),
\end{aligned}
$$

and

$$
\begin{aligned}
\mathcal{M} X^{[A} R_{3}^{B]}= & -2\left(W_{C}^{D} \mathcal{M}\right) X^{A}\left(W_{D}^{C} X^{B}\right)+\left(W_{C}^{C} \mathcal{M}\right) X^{A}\left(W_{D}^{D} X^{B}\right) \\
& +2\left(W_{C}^{D} \mathcal{M}\right) X^{B}\left(W_{D}^{C} X^{A}\right)-\left(W_{C}^{C} \mathcal{M}\right) X^{B}\left(W_{D}^{D} X^{A}\right) .
\end{aligned}
$$

Next, we consider $i \mathcal{M} \bar{\Psi}_{C} R_{1}^{A B C}$, whose explicit form is

$$
\begin{aligned}
i \mathcal{M} \bar{\Psi}_{C} R_{1}^{A B C}= & i \epsilon^{A B C D} \mathcal{M} \bar{\Psi}_{C}\left(X^{E} X_{E} \Psi_{D}-\Psi_{D} X_{E} X^{E}\right. \\
& \left.-2 X^{E} X_{D} \Psi_{E}+2 \Psi_{E} X_{D} X^{E}\right) .
\end{aligned}
$$

We construct a gauge variation $V_{C D}$, the actions of which on $X_{a}^{B \hat{a}}$ and $\bar{\Psi}_{B}$ are given by

$$
\left(V_{C D} X^{B}\right)_{a}^{\hat{a}} \equiv\left(\Psi_{C} X_{D}\right)_{\hat{b}}^{\hat{a}} X_{a}^{B \hat{b}}-X_{b}^{B \hat{a}}\left(X_{D} \Psi_{C}\right)_{a}^{b}
$$

and

$$
\left(V_{C D} \bar{\Psi}_{B}\right)_{a}^{\hat{a}} \equiv\left(\bar{\Psi}_{C} X_{D}\right)_{\hat{b}}^{\hat{a}} \Psi_{B a}^{\hat{b}}-\bar{\Psi}_{B b}^{\hat{a}}\left(X_{D} \Psi_{C}\right)_{a}^{b},
$$

respectively. $i \mathcal{M} \bar{\Psi}_{C} R_{1}^{A B C}$ could be written as

$$
\begin{aligned}
i \mathcal{M} \bar{\Psi}_{C} R_{1}^{A B C}= & i \epsilon^{A B C D} \mathcal{M} \bar{\Psi}_{C}\left[2\left(V_{E D} X^{E}\right)-\left(V_{\mathrm{DE}} X^{E}\right)\right] \\
= & i \epsilon^{A B C D} X^{E}\left[\bar{\Psi}_{C}\left(V_{\mathrm{DE}} \mathcal{M}\right)-2 \bar{\Psi}_{C}\left(V_{E D} \mathcal{M}\right)\right. \\
& \left.+\mathcal{M}\left(V_{\mathrm{DE}} \bar{\Psi}_{C}-2 V_{E D} \bar{\Psi}_{C}\right)\right]
\end{aligned}
$$

where

$$
\begin{aligned}
V_{\mathrm{DE}} \bar{\Psi}_{C}-2 V_{E D} \bar{\Psi}_{C} & =\bar{\Psi}_{D} X_{E} \Psi_{C}-\bar{\Psi}_{C} X_{E} \Psi_{D} \\
& -2 \bar{\Psi}_{E} X_{D} \Psi_{C}+2 \bar{\Psi}_{C} X_{D} \Psi_{E} .
\end{aligned}
$$

On the other hand,

$$
\begin{aligned}
\mathcal{M} X^{[A} R_{1}^{B]}= & 2 i \mathcal{M} X^{[A} \epsilon^{B] C D E} \bar{\Psi}_{C} X_{D} \Psi_{E} \\
= & i \mathcal{M} \epsilon^{A B C D} X^{E}\left(2 \bar{\Psi}_{E} X_{D} \Psi_{C}\right. \\
& \left.-2 \bar{\Psi}_{C} X_{D} \Psi_{E}+\bar{\Psi}_{C} X_{E} \Psi_{D}-\bar{\Psi}_{D} X_{E} \Psi_{C}\right) .
\end{aligned}
$$

So,

$$
\begin{aligned}
\mathcal{M} X^{[A} R_{1}^{B]}+i \mathcal{M} \bar{\Psi}_{C} R_{1}^{A B C}= & i \epsilon^{A B C D} X^{E}\left[\bar{\Psi}_{C}\left(V_{\mathrm{DE}} \mathcal{M}\right)\right. \\
& \left.-2 \bar{\Psi}_{C}\left(V_{E D} \mathcal{M}\right)\right]
\end{aligned}
$$

Finally, we consider $\mathcal{M} X^{[A} R_{2}^{B]}$. The actions of the gauge variation $U^{C B}$ on $\Psi_{A a}^{\hat{a}}$ and $X_{a}^{A \hat{a}}$ are defined as

$$
\left(U^{C B} \Psi_{A}\right)_{a}^{\hat{a}} \equiv\left(X^{C} \bar{\Psi}^{B}\right)_{\hat{b}}^{\hat{a}} \Psi_{A a}^{\hat{b}}-\bar{\Psi}_{A b}^{\hat{a}}\left(\Psi^{B} X^{C}\right)_{a}^{b}
$$

and

$$
\left(U^{C B} X^{A}\right)_{a}^{\hat{a}} \equiv\left(X^{C} \bar{\Psi} B\right)_{\hat{b}}^{\hat{a}} X_{a}^{A \hat{b}}-X_{b}^{A \hat{a}}\left(\bar{\Psi}^{B} X^{C}\right)_{a}^{b}
$$

respectively. Then,

$$
R_{2}^{B}=i\left[2\left(U^{C B} \Psi_{C}\right)-\left(U^{B C} \Psi_{C}\right)\right] .
$$

$\mathcal{M} X^{[A} R_{2}^{B]}$ becomes

$$
\begin{aligned}
\mathcal{M} X^{[A} R_{2}^{B]}= & i \mathcal{M}\left\{X^{A}\left[2\left(U^{C B} \Psi_{C}\right)-\left(U^{B C} \Psi_{C}\right)\right]-X^{B}\left[2\left(U^{C A} \Psi_{C}\right)-\left(U^{A C} \Psi_{C}\right)\right]\right\} \\
= & i\left[\left(U^{B C} \mathcal{M}\right) X^{A}-2\left(U^{C B} \mathcal{M}\right) X^{A}+2\left(U^{C A} \mathcal{M}\right) X^{B}-\left(U^{A C} \mathcal{M}\right) X^{B}\right] \Psi_{C} \\
& +i \mathcal{M}\left[\left(U^{B C} X^{A}\right)-\left(U^{A C} X^{B}\right)+2\left(U^{C A} X^{B}\right)-2\left(U^{C B} X^{A}\right)\right] \Psi_{C}
\end{aligned}
$$

where

$$
\begin{aligned}
& \left(U^{B C} X^{A}\right)-\left(U^{A C} X^{B}\right)+2\left(U^{C A} X^{B}\right)-2\left(U^{C B} X^{A}\right) \\
& \quad=2\left(X^{B} \bar{\Psi}^{C} X^{A}-X^{A} \bar{\Psi}^{C} X^{B}+X^{C} \bar{\Psi}^{A} X^{B}-X^{B} \bar{\Psi}^{A} X^{C}-X^{C} \bar{\Psi}^{B} X^{A}+X^{A} \bar{\Psi}^{B} X^{C}\right)
\end{aligned}
$$

On the other hand, 


$$
\begin{aligned}
i \mathcal{M} \bar{\Psi}_{C} R_{2}^{A B C} & =2 i \mathcal{M} \bar{\Psi}_{C}\left(X^{A} \Psi^{C} X^{B}-X^{A} \Psi^{B} X^{C}+X^{B} \Psi^{A} X^{C}-X^{B} \Psi^{C} X^{A}+X^{C} \Psi^{B} X^{A}-X^{C} \Psi^{A} X^{B}\right) \\
& =2 i \mathcal{M}\left(X^{A} \bar{\Psi}^{C} X^{B}-X^{A} \bar{\Psi}^{B} X^{C}+X^{B} \bar{\Psi}^{A} X^{C}-X^{B} \bar{\Psi}^{C} X^{A}+X^{C} \bar{\Psi}^{B} X^{A}-X^{C} \bar{\Psi}^{A} X^{B}\right) \Psi_{C}
\end{aligned}
$$

We get

$$
\mathcal{M} X^{[A} R_{2}^{B]}+i \mathcal{M} \bar{\Psi}_{C} R_{2}^{A B C}=i\left[\left(U^{B C} \mathcal{M}\right) X^{A}-2\left(U^{C B} \mathcal{M}\right) X^{A}+2\left(U^{C A} \mathcal{M}\right) X^{B}-\left(U^{A C} \mathcal{M}\right) X^{B}\right] \Psi_{C}
$$

With Eqs. (B9), (B15), and (B22) combined together,

$$
\begin{aligned}
\mathcal{M} & \left(X^{[A} R_{1}^{B]}+X^{[A} R_{2}^{B]}+X^{[A} R_{3}^{B]}\right)+i \mathcal{M} \bar{\Psi}_{C}\left(R_{1}^{A B C}+R_{2}^{A B C}\right) \\
= & 2\left(W_{C}^{D} \mathcal{M}\right) X^{B}\left(W_{D}^{C} X^{A}\right)-2\left(W_{C}^{D} \mathcal{M}\right) X^{A}\left(W_{D}^{C} X^{B}\right)+\left(W_{C}^{C} \mathcal{M}\right) X^{A}\left(W_{D}^{D} X^{B}\right) \\
& -\left(W_{C}^{C} \mathcal{M}\right) X^{B}\left(W_{D}^{D} X^{A}\right)+i \epsilon^{A B C D} X^{E} \bar{\Psi}_{C}\left(V_{D E} \mathcal{M}\right)-2 i \epsilon^{A B C D} X^{E} \bar{\Psi}_{C}\left(V_{E D} \mathcal{M}\right) \\
& +i\left(U^{B C} \mathcal{M}\right) X^{A} \Psi_{C}-2 i\left(U^{C B} \mathcal{M}\right) X^{A} \Psi_{C}+2 i\left(U^{C A} \mathcal{M}\right) X^{B} \Psi_{C}-i\left(U^{A C} \mathcal{M}\right) X^{B} \Psi_{C}
\end{aligned}
$$

The conservation condition for $j_{\mu}^{A B}$ is

$$
\begin{aligned}
\partial^{\mu} j_{\mu}^{A B}= & -\frac{i k}{4 \pi}\left(D^{\mu} \mathcal{M}\right)\left[X^{A}\left(D_{\mu} X^{B}\right)-\left(D_{\mu} X^{A}\right) X^{B}+\frac{i}{2} \epsilon^{A B C D} \bar{\Psi}_{C} \gamma_{\mu} \Psi_{D}\right] \\
& -\left(W_{C}^{D} \mathcal{M}\right)\left[X^{B}\left(W_{D}^{C} X^{A}\right)-X^{A}\left(W_{D}^{C} X^{B}\right)\right]-\frac{1}{2}\left(W_{C}^{C} \mathcal{M}\right)\left[X^{A}\left(W_{D}^{D} X^{B}\right)-X^{B}\left(W_{D}^{D} X^{A}\right)\right] \\
& -\frac{i}{2} \bar{\Psi}_{C}\left(V_{D E} \mathcal{M}\right)\left(\epsilon^{A B C D} X^{E}-2 \epsilon^{A B C E} X^{D}\right) \\
& -\frac{i}{2}\left[\left(U^{B C} \mathcal{M}\right) X^{A}-\left(U^{A C} \mathcal{M}\right) X^{B}+2\left(U^{C A} \mathcal{M}\right) X^{B}-2\left(U^{C B} \mathcal{M}\right) X^{A}\right] \Psi_{C} \\
= & 0 .
\end{aligned}
$$

[1] G. 't Hooft, On the phase transition towards permanent quark confinement, Nucl. Phys. B138, 1 (1978).

[2] V. Borokhov, A. Kapustin, and X. Wu, Topological disorder operators in three-dimensional conformal field theory, J. High Energy Phys. 11 (2002) 049.

[3] K. A. Intriligator and N. Seiberg, Mirror symmetry in three dimensional gauge theories, Phys. Lett. B 387, 513 (1996).

[4] V. Borokhov, A. Kapustin, and X. Wu, Monopole operators and mirror symmetry in three-dimensions, J. High Energy Phys. 12 (2002) 044.

[5] V. Borokhov, Monopole operators in three-dimensional $\mathcal{N}=4 \mathrm{SYM}$ and mirror symmetry, J. High Energy Phys. 03 (2004) 008.

[6] D. Bashkirov, Aharony duality and monopole operators in three dimensions, arXiv:1106.4110.

[7] O. Aharony, S. S. Razamat, N. Seiberg, and B. Willett, 3D dualities from 4D dualities, J. High Energy Phys. 07 (2013) 149.

[8] K. Intriligator, Matching 3D $\mathcal{N}=2$ vortices and monopole operators, J. High Energy Phys. 10 (2014) 52.
[9] O. Aharony, Baryons, monopoles and dualities in ChernSimons-matter theories, J. High Energy Phys. 02 (2016) 093.

[10] S. R. Coleman, Quantum Sine-Gordon equation as the massive thirring model, Phys. Rev. D 11, 2088 (1975).

[11] S. Mandelstam, Soliton operators for the quantized SineGordon equation, Phys. Rev. D 11, 3026 (1975).

[12] O. Aharony, O. Bergman, D. L. Jafferis, and J. Maldacena, $\mathcal{N}=6$ superconformal Chern-Simons-matter theories, M2branes and their gravity duals, J. High Energy Phys. 10 (2008) 091.

[13] D. Berenstein and D. Trancanelli, Three-dimensional $\mathcal{N}=$ 6 SCFT's and their membrane dynamics, Phys. Rev. D 78, 106009 (2008).

[14] I. Klebanov, T. Klose, and A. Murugan, $\mathrm{AdS}_{4} / \mathrm{CFT}_{3}$ squashed, stretched and warped, J. High Energy Phys. 03 (2009) 140.

[15] Y. Imamura and S. Yokoyama, $\mathcal{N}=4$ Chern-Simons theories and wrapped M-branes in their gravity duals, Prog. Theor. Phys. 121, 915 (2009). 
[16] Y. Imamura, Monopole operators in $\mathcal{N}=4$ Chern-Simons theories and wrapped M2-branes, Prog. Theor. Phys. 121, 1173 (2009).

[17] I. R. Klebanov, S. S. Pufu, and F. D. Rocha, The squashed, stretched, and warped gets perturbed, J. High Energy Phys. 06 (2009) 019.

[18] M. M. Sheikh-Jabbari and J. Simon, On half-BPS states of the ABJM theory, J. High Energy Phys. 08 (2009) 073.

[19] D. Berenstein and J. Park, The BPS spectrum of monopole operators in ABJM: Towards a field theory description of the giant torus, J. High Energy Phys. 06 (2010) 073.

[20] I. R. Klebanov, S. S. Pufu, and T. Tesileanu, Membranes with topological charge and $\mathrm{AdS}_{4} / \mathrm{CFT}_{3}$ correspondence, Phys. Rev. D 81, 125011 (2010).

[21] M. Cesàro, G. Larios, and O. Varela, A cubic deformation of ABJM: The squashed, stretched, warped, and perturbed gets invaded, J. High Energy Phys. 10 (2020) 041.

[22] S. Kim, The complete superconformal index for $\mathcal{N}=6$ Chern-Simons theory, Nucl. Phys. B821, 241 (2009).

[23] D. Bashkirov and A. Kapustin, Supersymmetry enhancement by monopole operators, J. High Energy Phys. 05 (2011) 015.

[24] A. Gustavsson and S. J. Rey, Enhanced $\mathcal{N}=8$ Supersymmetry of ABJM Theory on $R(8)$ and $R(8) / Z(2)$, arXiv:0906.3568.

[25] O. K. Kwon, P. Oh, and J. Sohn, Notes on supersymmetry enhancement of ABJM theory, J. High Energy Phys. 08 (2009) 093.

[26] H. Samtleben and R. Wimmer, $\mathcal{N}=6$ Superspace Constraints, SUSY enhancement and monopole operators, J. High Energy Phys. 10 (2010) 080.

[27] P. Goddard, J. Nuyts, and D. I. Olive, Gauge theories and magnetic charge, Nucl. Phys. B125, 1 (1977).

[28] M. K. Benna, I. R. Klebanov, and T. Klose, Charges of monopole operators in Chern-Simons Yang-Mills theory, J. High Energy Phys. 01 (2010) 110.

[29] G. W. Moore and N. Seiberg, Taming the conformal zoo, Phys. Lett. B 220, 422 (1989).

[30] K. Gawedzki, Boundary WZW, G/H, G/G and CS theories, Ann. Inst. Henri Poincaré 3, 847 (2002).
[31] N. Itzhaki, Anyons, 't Hooft loops and a generalized connection in three dimensions, Phys. Rev. D 67, 065008 (2003).

[32] C. Cordova, T. T. Dumitrescu, and K. Intriligator, Multiplets of superconformal symmetry in diverse dimensions, J. High Energy Phys. 03 (2019) 163.

[33] G. V. Dunne, R. Jackiw, and C. A. Trugenberger, ChernSimons theory in the Schrödinger representation, Ann. Phys. (Berlin) 194, 197 (1989).

[34] M. A. Bandres, A. E. Lipstein, and J. H. Schwarz, Studies of the ABJM theory in a formulation with manifest SU(4) R-symmetry, J. High Energy Phys. 09 (2008) 027.

[35] I. R. Klebanov and G. Torri, M2-branes and AdS/CFT, Int. J. Mod. Phys. A 25, 332 (2010).

[36] M. F. Sohnius, Bianchi identities for supersymmetric gauge theories, Nucl. Phys. B136, 461 (1978).

[37] J. Maldacena and A. Zhiboedov, Constraining conformal field theories with a higher spin symmetry, J. Phys. A 46, 214011 (2013).

[38] N. Lambert, C. Papageorgakis, and M. Schmidt-Sommerfeld, Instanton operators in five-dimensional gauge theories, J. High Energy Phys. 03 (2015) 019.

[39] D. Rodriguez-Gomez and J. Schmude, Supersymmetrizing 5D instanton operators, J. High Energy Phys. 03 (2015) 114.

[40] O. Bergman and D. Rodriguez-Gomez, A note on instanton operators, instanton particles, and supersymmetry, J. High Energy Phys. 05 (2016) 068.

[41] N. Seiberg, Five-dimensional SUSY field theories, nontrivial fixed points and string dynamics, Phys. Lett. B 388, 753 (1996).

[42] D. Bashkirov, A comment on the enhancement of global symmetries in superconformal $\mathrm{SU}(2)$ gauge theories in $5 \mathrm{D}$, arXiv:1211.4886.

[43] Y. Tachikawa, Instanton operators and symmetry enhancement in 5D supersymmetric gauge theories, Prog. Theor. Exp. Phys. 2015, 043 B06 (2015).

[44] G. Zafrir, Instanton operators and symmetry enhancement in 5D supersymmetric USp, SO and exceptional gauge theories, J. High Energy Phys. 07 (2015) 087.

[45] K. Yonekura, Instanton operators and symmetry enhancement in 5D supersymmetric quiver gauge theories, J. High Energy Phys. 07 (2015) 167. 